\title{
LOCALISATION ET INTERCULTUREL
}

\author{
Présenté par \\ Rim Hafez ELBAKARY \\ Maître de conférences \\ FILIERE DES LANGUES APPLIQUEES
}

مجلة الدراسات التربوية والانساتية ـ كلية التربية ـ جامعة دمنهور

المجلد الثانى عشر - العدد الرآبع - الجزع الثالث - لسنة 2020 
مجلة الدراسات التربوية والانسانية ـ كلية التربية ـ جامعة دمنهور ـ المجلد الثانى عثر - العدد الرابع - الجزء الثالث - لسنة 2020

\section{1}




\section{LOCALISATION ET INTERCULTUREL \\ Rim Hafez ELBAKARY}

\section{Résumé :}

La localisation, est une discipline récente, qui fait partie de la traduction multimédias et est inhérente à la technologie. Une bonne localisation d'un produit contribue, comme toute forme de communication, à abattre toute barrière culturelle pouvant se dresser entre la langue source et la langue cible; ou encore à diminuer l'écart culturel pouvant exister entre la culture de départ et celle d'arrivée. Le travail du localisateur s'avère donc indispensable pour une bonne réception, commercialisation et acceptation du produit dans la société cible ainsi que pour un bon dialogue interculturel. Dans cette étude, nous allons donc commencer par définir la localisation, suivre son évolution et souligner le rôle joué par la traduction comme premier élément dans la communication interculturelle ; nous allons, ensuite, essayer de cerner de plus près les divergences et les convergences pouvant exister entre la traduction et la localisation pour arriver à déceler les différents rôles que joue cette nouvelle forme de traduction-adaptation dans la communication interculturelle. Nous expliquerons, ensuite, le processus de la localisation - comme étant l'un des types de traduction qui présente un nombre de défis résolument actuels, façonnés par la mondialisation et l'évolution des nouvelles technologies. Nous allons enfin, en adoptant une approche comparative comme nous venons de le mentionner plus haut, passer en revue les différents types de localisation avec des exemples de produits localisés à l'appui.

Mots clés :

Localisation-adaptation-interculturel-communication. 


\section{Introduction :}

A l'ère du numérique et de la mondialisation, la traduction, comme toute autre discipline commence à prendre de l'élan et revêtir une allure différente : on parle alors, entre autres, de "traduction multimédia" qui porte sur les domaines du soustitrage, du doublage/synchronisation, des commentaires, et de la localisation ${ }^{1}$.

En fait, ces différents visages de la traduction multimédia, tous inhérents à la technologie, sont basés sur un processus d'assimilation ou d'appropriation qui rend possible la présentation de l'étranger conformément aux besoins de la culture cible. L'émergence de nouveaux métiers liés au processus de traduction voit alors le jour : on ne parle plus de traducteur littéraire ou spécialisé uniquement, mais de traducteur-audiovisuel, de traducteur-localisateur ou d'adaptateur multimédias. Ces nouveaux métiers qui combinent traduction et technique impliquent donc de la part du traducteur, de la polyvalence et un goût prononcé pour l'informatique.

Or, si maintes études ont été faites sur la traduction audiovisuelle (sous-titrage,

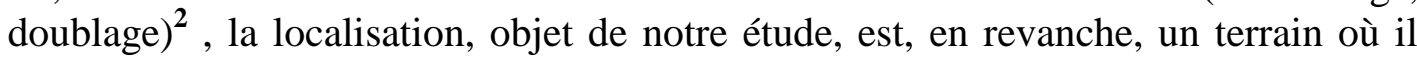
reste encore tant à défricher. Et les recherches :

«... menées dans ce domaine spécialisé de la traduction, nous permettent d'observer que la plupart des études sur la localisation suivent une approche purement technique ou économique. De même que très peu de spécialistes en localisation se consacrent à l'enseignement, très peu de chercheurs s'investissent dans des études sur les divers aspects de la localisation en tant qu'activité de traduction, ce qui semble étonnant au

${ }^{1}$ Cf. infra p.4 pour la définition détaillée de la localisation.

- $\quad{ }^{2}$ Les études sont nombreuses, citons à titre d'exemple : l'ouvrage de Jean-François CORNU : Le doublage et le sous- titrage : Histoire et esthétique, 2014. L'ouvrage de Thierry LE NOUVEL : Le Doublage, Eyrolles, Ciné-métiers, 2007 et la thèse de Zoé PETTIT, Sous titrage et doublage : interaction entre langage et image dans une sélection de genres audiovisuels, soutenue à Montpellier 3, sous la direction de Jacky MARTIN, 2000. 
regard des enjeux académiques et économiques concernés $»^{3}$.

Pour combler ce manque dans les recherches dédiées à la localisation, et prouver qu'une bonne localisation d'un produit contribue, comme toute forme de communication, à abattre toute barrière culturelle pouvant se dresser entre la langue source et la langue cible ; ou encore à diminuer l'écart culturel pouvant exister entre la culture de départ et celle d'arrivée, nous avons décidé d'entreprendre cette étude. L'idée de cette étude nous a été également inspirée par un stage " Formation de formateurs en localisation » que nous avons suivi au sein de l'entreprise Arabize ${ }^{4}$ pour un mois, en Août 2015. Nous avons voulu, par cette étude, partager l'expérience que nous avons acquise au cours de ce stage avec les jeunes chercheurs dans le domaine de la localisation, afin de les munir, à travers les différents exemples que nous avons choisis, des outils nécessaires qui leur permettront d'avoir un regard critique sur tout produit localisé et de leur expliquer comment le travail du localisateur s'avère indispensable pour une bonne réception, commercialisation et acceptation du produit dans la société cible .

Ainsi, dans la présente étude, nous allons adopter une approche comparative en nous basant sur des produits étrangers localisés pour l'Egypte et inversement pour montrer comment le travail du localisateur sert à tisser des liens de compréhension et de tolérance entre une société source et une société cible; et pour souligner l'importance de la localisation dans la création d'un véritable dialogue interculturel.

Nous allons donc commencer par définir la localisation, suivre son évolution et souligner le rôle joué par la traduction comme premier élément dans la communication interculturelle; nous allons, ensuite, essayer de cerner de plus près les divergences et les convergences pouvant exister entre la traduction et la localisation pour arriver à déceler les différents rôles que joue cette nouvelle forme de traductionadaptation dans la communication interculturelle. Nous expliquerons, ensuite, le processus de la localisation - comme étant l'un des types de

\footnotetext{
${ }^{3}$ Ma'moun ALSHTAIWI, «Parlons du localisateur : rôle et profil », in Traduire, n. 237, 2017, pp.38-47.

${ }^{4}$ Cf. Infra. p. 6. pour des informations plus détaillées concernant l'entreprise Arabize.
} 
traduction qui présente un nombre de défis résolument actuels, façonnés par la mondialisation et l'évolution des nouvelles technologies. Nous allons enfin, en adoptant une approche comparative comme nous venons de le mentionner plus haut, passer en revue les différents types de localisation avec des exemples de produits localisés à l'appui.

\section{Définition et évolution de la localisation :}

La localisation, cette industrie très récente, a connu plusieurs définitions à travers les années. En effet, étymologiquement, le mot « localisation » est un mot emprunté à l'anglais «locale » qui renvoie initialement à l'idée d'un lieu spécifique ${ }^{5}$, lequel avec l'émergence et le développement de la technologie, acquiert un sens plus large. Ainsi, vers les années 80, la localisation portait uniquement sur la traduction des logiciels en vue de leur diffusion sur un marché spécifique, d'où la définition que nous donne ESSELINK: "Localization is the translation and adaptation of a software or web product, which includes the software application itself and all related product documentation $»^{6}$. LISA $^{7}$ propose presque la même définition considérant la localisation comme : «The process of modifying products or services to account for differences in distinct markets. In practice, this means that localization needs to address three main categories of issues: linguistic issues, content and cultural issues and technical issues".

À la définition, qui était alors donnée par ESSELINK et la LISA, s'ajoutent aussi d'autres définitions. La localisation est, selon Le Grand Dictionnaire de l'Office Québécois de La Langue Française, l'«adaptation sur le plan linguistique et culturel des logiciels et de la documentation qui les accompagne en vue de leur diffusion sur un marché spécifique »" Elle est, également, selon le

\footnotetext{
${ }^{5}$ Bert ESSELINK, A practical guide to localization, Amsterdam/Philadelphia, John Benjamins Publishing Company, 2000,

p.11.

${ }^{6}$ Ibid., p.12.

Notre traduction : La localisation est la traduction et l'adaptation d'un logiciel ou d'un produit Web, qui comprend l'application logicielle elle-même et toute la documentation qui lui est associée.

${ }^{7}$ LISA : The Localization Industry Standards Association, Fondée en 1990 en Suisse, est une association privée à but non-lucratif. LISA fut l'organisation de premier plan pour l'industrie de la localisation et de l'internationalisation. Elle ferma ses portes en février 2011.

${ }^{8}$ Notre traduction: Le processus de modification de produits ou de services tient ainsi compte des différences dans des marchés diverses. En pratique, cela signifie que la localisation doit aborder trois grandes catégories : linguistiques, culturelles et techniques.

${ }^{9} \mathrm{http} / / / \mathrm{www}$.granddictionnaire.com/ficheOqlf.aspx?Id_Fiche=506326

Dernière consultation le 5 avril 2018
}

505 
Larousse : «l'adaptation d'un produit, d'une activité productrice ou commerciale à une zone géographique, en fonction de divers facteurs naturels, techniques, économiques, culturels et sociaux $\gg{ }^{10}$.

Quant à l'industrie de la localisation, celle-ci est relativement jeune puisqu'elle date des années 1980 lorsque les éditeurs de logiciels, basés aux États-Unis, réalisent, à la suite de la croissance d'Internet, que la traduction et l'adaptation des produits peuvent commercialiser et distribuer leurs produits dans d'autres pays et par conséquent, augmenter leur profit.

La plupart de ces éditeurs commence par créer au sein de leurs entreprises un département de traduction, pour localiser leurs produits. Mais, avec l'accroissement du nombre de projets, ils réalisent qu'ils ne possèdent ni les moyens, ni le temps, ni les compétences nécessaires pour gérer ce processus innovant de localisation. C'est ainsi que les premières sociétés spécialisées dans les projets multilingues de traduction et de localisation vont voir le jour au début des années 90.

Ces sociétés spécialisées commenceront par offrir non seulement le service de traduction, mais aussi l'ingénierie, la publication assistée par ordinateur et les tests de qualité. C'est donc pendant cette période que la distinction entre traduction et localisation commença.

Berceau de l'industrie de la localisation, L'Irlande, s'est affirmée depuis le début en tant que pays leader dans cette branche, grâce aux efforts et aux investissements du gouvernement. En 2002, GALA (Globalization and Localization Association) ${ }^{11}$ voit le jour avec 15 entreprises comme membres. Étant une association mondiale à but non lucratif, elle soutient ses membres en partageant le savoir et en faisant progresser la technologie.

La localisation ne s'arrête pas en Occident mais franchit les frontières du monde entier et arrive jusqu'en Egypte et aux pays du Golfe en raison de la croissance considérable des besoins et de l'ouverture du marché arabe à l'international. Ainsi, et dans le but d'attirer les investissements et d'accroître les échanges commerciaux, de multiples agences et entreprises spécialisées dans le domaine de la localisation furent créées. Ces dernières proposent leurs services qui localisent soit des produits arabes pour un marché étranger, soit des produits étrangers pour un marché arabe.

\section{La localisation en Egypte :}

En Egypte, tout particulièrement, le marché effectif de la localisation s'avère très concurrentiel. Il a connu un essor considérable depuis la fin des années 90 avec

\footnotetext{
${ }^{10} \mathrm{https}: / / \mathrm{www}$. larousse.fr/dictionnaires/francais/localisation/47591?q=la+localisation\#47516 Dernière consultation le 7 avril 2018

${ }^{11} \mathrm{https}: / / \mathrm{www}$. gala-global.org/

Dernière consultation le 10/10/2018
} 
environ une quarantaine d'agences spécialisées, connues sur le plan national et international, dont cinq sont membres de l'association GALA, parmi lesquelles on peut citer à titre d'exemple: E-Localize ${ }^{12}$, Skylingos $^{13}$, ARABIZATION AND TRANSLATION $^{14}$, et Arabize ${ }^{15}$.

Ces agences, comme leurs semblables dans le monde entier, ont compris le besoin accru «des grands canons de la mondialisation d'intégrer la spécificité culturelle locale, dans la stratégie promotionnelle internationale de leurs produits ${ }^{16}$. Les services qu'elles proposent rejoignent donc cet objectif, qu'il s'agisse de localiser des produits arabes pour un marché étranger ou bien de localiser un produit étranger pour un marché arabe.

Arabize $^{17}$ est une entreprise leader dans la localisation arabe, la documentation et le développement de contenu. Fondée en 1994, par son PDG Manal Amin accompagnée d'une équipe de 30 employés, Arabize compte actuellement plus de 100 employés. Au moment où Arabize a été fondée, rares étaient les institutions qui donnaient une importance à la langue arabe, ce qui lui valut sa réputation de pionnière dans ce domaine. Avec le développement de la technologie et des services Internet, ses concurrents se multiplièrent sur le marché ainsi que ses efforts pour maintenir son image.

Les services d'Arabize ont commencé par la localisation et la traduction; ensuite ont progressé pour inclure le développement et la gestion de contenu, les tests linguistiques et l'éditique. Notons que le fait d'avoir compris les normes de la localisation, a permis à Arabize, dès sa création, d'être le premier choix des entreprises multinationales comme Oracle, SAP, Lotus, Novell, IBM, Microsoft, Google. Grâce à la

12 https://www.elocalize.net/

Dernière consultation le 12/10/2018

${ }^{13} \mathrm{http}: / /$ skylingos.com/

Dernière consultation le 12/10/2018

${ }^{14}$ https://arabization-translation.net/

Dernière consultation le 12/10/2018

${ }^{15} \mathrm{http}: / /$ www.arabize.com/

Dernière consultation le 12/10/2018

${ }^{16}$ Paula Bouffard et Philippe Caignon, «Localisation et variation linguistique. Vers une géolinguistique de l'espace virtuel francophone », in Meta, Volume 51, Numéro 4, décembre 2006, pp. 806-823.

${ }^{17}$ Cf. infra p.7 pour l'interface du site d'Arabize

507 
qualité de ses services, Arabize sera certifiée par l'Organisation internationale de normalisation, et obtiendra ainsi l'ISO officielle 9001 : 2000 en 2005, ISO $9001: 2008$ en 2008 et EN $15038: 2006$ Certification en $2009^{18}$.

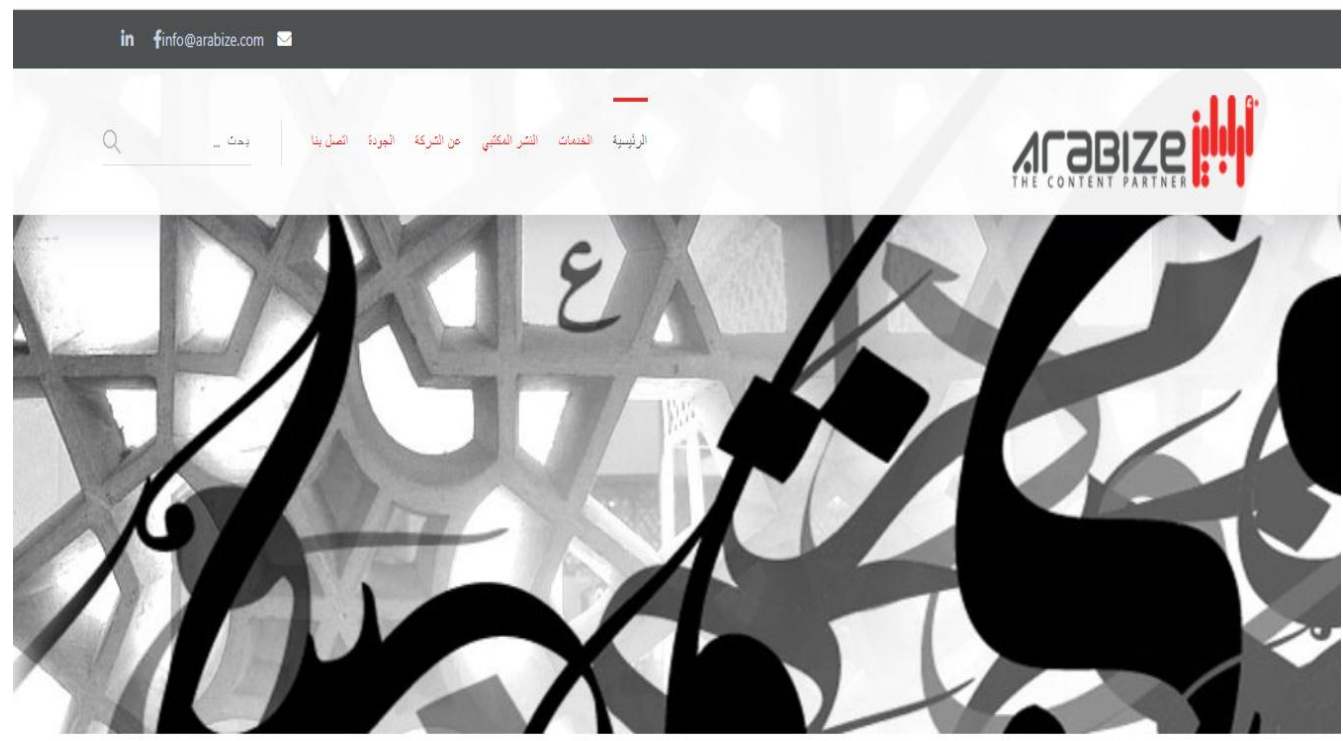

\section{Figure ( 1 ) Interface du site d'Arabize}

E-Localize ${ }^{19}$, a été fondée en 2000 en tant que filiale de la compagnie North Africa pour la technologie (NAC), l'un des plus grands fournisseurs de matériel informatique dans la région du Moyen-Orient. E-Localize offre une gamme complète de services de localisation de sites internet, d'interfaces graphiques, de fichiers d'aide, de logiciels, de documentation technique, et de matériel de marketing. Tous ses traducteurs ont une formation universitaire spécialisée dans leurs domaines. Les traducteurs indépendants sont soigneusement testés et régulièrement évalués. Son équipe d'ingénieurs peut résoudre toute forme de problèmes : conversion, insertion ou récupération de données. Ils sont indispensables pour la localisation. Son studio de $\mathrm{PAO}^{20}$ peut fournir des solutions

${ }^{18} \mathrm{Cf}$. http://www.arabize.com/

Notre traduction du contenu anglais présent sur le site d'Arabize.

${ }^{19}$ Cf. infra p.8 pour l'interface du site de E-Localize.

-

${ }^{20}$ La PAO (Publication Assistée par Ordinateur) est entrée en vigueur milieu des années 80 et s'est développée à partir des années 90 . Une formation PAO complète 
de qualité prêtes à l'impression de toutes tailles, dans toutes les langues et polices d'écriture 21

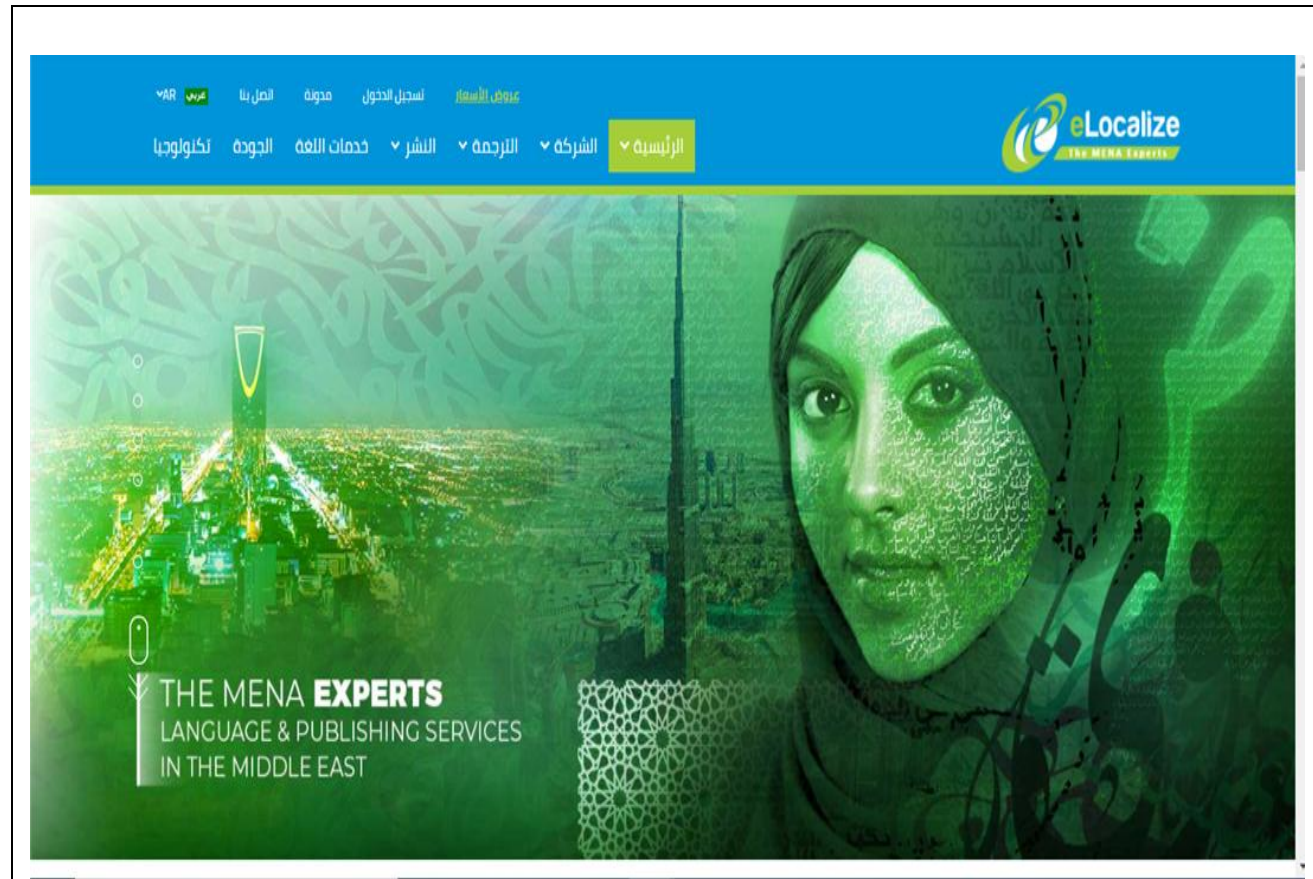

Figure ( 2 )Interface du site de E-Localize

Créée en 2010, ARABIZATION and TRANSLATION ${ }^{22}$ est le site bilingue de Noha Mostafa, qui est une traductrice égyptienne spécialisée dans la traduction anglaise / arabe / anglaise, offrant une traduction certifiée et professionnelle à des organismes officiels, des ministères, des ambassades, des associations et des individus. Le site web de ARABIZATION and TRANSLATION.

Sa gamme de services comprend la traduction, l'édition, la relecture, la transcription, la localisation (des sites Web, des logiciels, des livrets d'apprentissage

doit comprendre la formation à un logiciel de mise en page ( InDesign ou Quark XPress), un logiciel qui traite l'image pixel (Photoshop) et un logiciel qui traite l'image vectorielle (Illustrator).

https://www.formation-graphisme-marseille.com/pao/

Dernière consultation le 26 mai 2019

${ }^{21}$ Notre traduction de la version arabe de la rubrique 'Qui sommes-nous ?'”présente sur le site officiel de E-Localize.

http://www.elocalize.net/ar-EG/Company/about-us

Dernière consultation le 27 mai 2019

${ }^{22}$ Cf. infra.p.9 pour l'interface du site ARABIZATION and TRANSLATION

509 
électronique et de matériels pédagogiques), le sous-titrage, l'adaptation et la transcréation, la traduction-instruction, la formation PAO et la composition. ARABIZATION and TRANSLATION a, de plus, un privilège important : il adapte linguistiquement sa traduction. Ainsi, il traduit en arabe classique (utilisé dans la région du Moyen-Orient) et en arabe moderne (utilisé dans tous les pays arabes); et pour l'anglais, il traduit en anglais britannique et en anglais américain ${ }^{23}$.

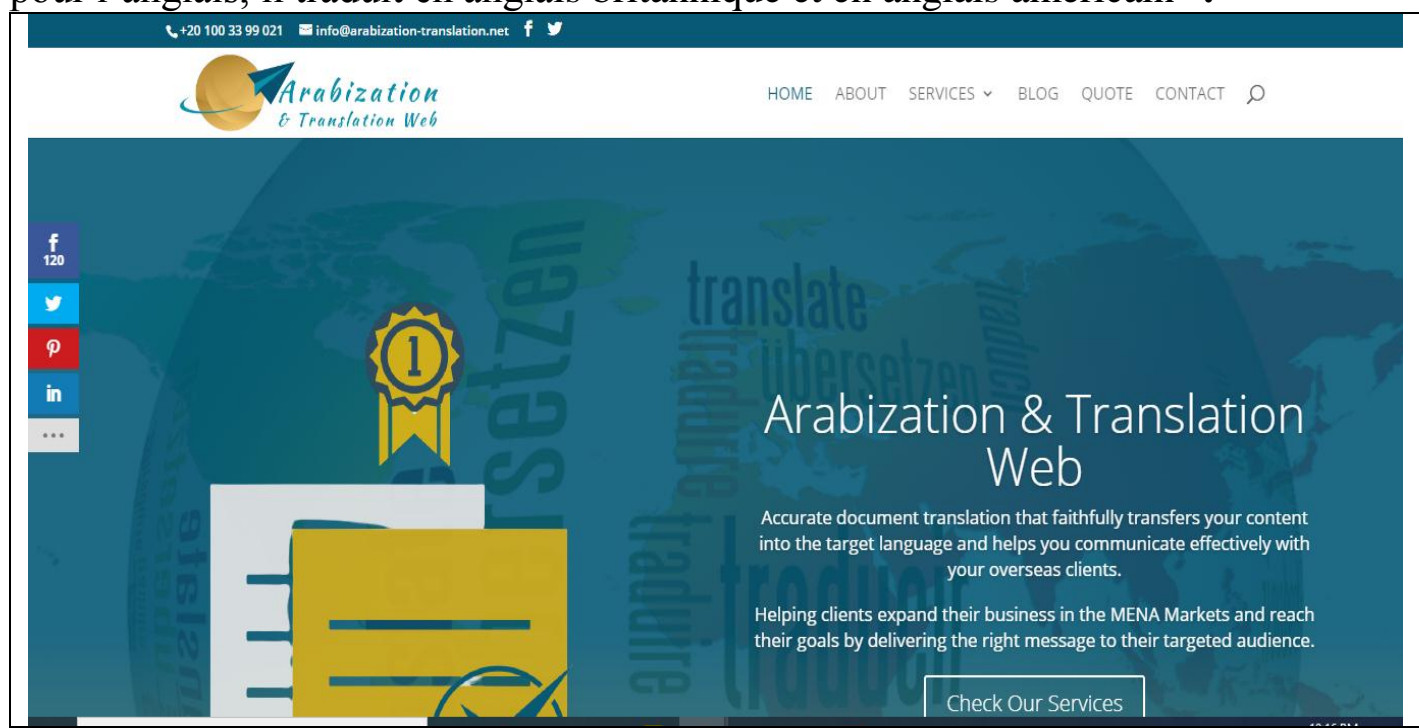

Figure ( 3 ) Interface du site de ARABIZATION and TRANSLATION

SkyLingos ${ }^{24}$ est une autre grande entreprise de localisation, créée en 2012, dont le siège principal se trouve au Caire. Cette entreprise offre un large éventail de services linguistiques et de sciences de la vie, à savoir la traduction, la localisation, les tests, le DTP, la vie, les affaires, les RH et les compétences techniques. Le fondateur de SkyLingos a toujours proposé la traduction clé en anglais / arabe et vice-versa, la localisation, la révision, les services de création de contenu pour les entreprises leaders de l'industrie de la technologie, des logiciels et des industries de l'édition. Bien davantage, SkyLingos ne se restreint pas à jouer le rôle de fournisseur de contenu, mais offre des services de formation, de consultation, des services linguistiques adaptés aux besoins des clients, à leurs horaires, ainsi qu'à leur budget ${ }^{25}$.

${ }^{23}$ Notre traduction de la version anglaise du site ARABIZATION and TRANSLATION

${ }^{24}$ Cf. infra.p. 10 pour l'interface du site SkyLingos

${ }^{25}$ Notre traduction de certaines informations à propos du site présentes dans la version anglaise de SkyLingos.

Cf. http://skylingos.com/ 
L'Egypte est donc considérée comme un marché propice pour la localisation sous ses différents aspects, et par conséquent, un champ idéal offrant de grandes opportunités de travail à tous ceux qui s'intéressent à la traduction en particulier et à la technologie, qui lui est inhérente, en général.

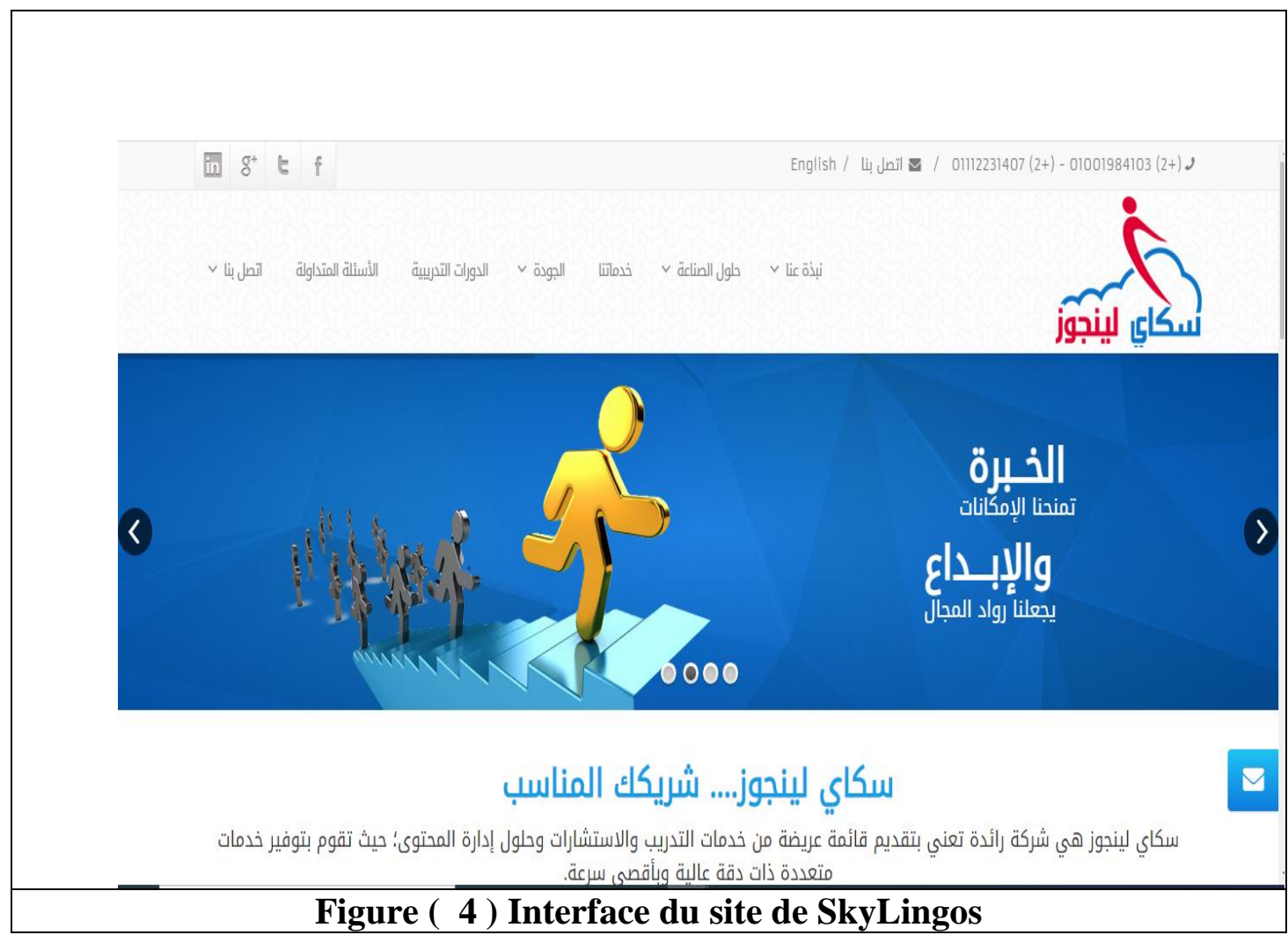

3.La localisation et la langue arabe : un marché en plein essor : La langue arabe est, dans notre cas, la langue vers laquelle sera localisé un produit. Et comme cette langue a été adoptée «comme l'une des six langues officielles des Nations Unies, rejoignant le chinois, l'anglais, le français, le russe et l'espagnol $\gg^{26}$ - langue officielle dans 26 pays et langue parlée par des millions de musulmans dans d'autres pays du monde $^{27}$ - le travail de localisation s'avère donc important vu le nombre

\footnotetext{
${ }^{26} \mathrm{https}$ ://www.lescahiersdelislam.fr/Histoire-et-evolution-de-la-langue-arabe a137.html Dernière consultation le 08/08/2019

${ }^{27}$ Cf. Ibid. Loc.cit

511
} 
considérable d'utilisateurs arabophones présents sur la toile ou consommateurs de différents produits étrangers. Ajoutons également que les vastes perspectives commerciales dans les pays producteurs de pétrole, qui constituent à l'heure actuelle, les marchés économiquement les plus prometteurs, ont entraîné un accroissement du volume des traductions professionnelles pour l'arabe.

Or, ce travail de localisation a commencé dans le monde arabe depuis bien longtemps et précisément depuis le Moyen Age, mais sous l'appellation de «traduction». On renvoie ici, en particulier, à la première période de la dynastie abbasside (750-905) ; car c'est dès cette époque que la traduction devint une activité florissante dans le monde islamique. Celle-ci a joué un rôle capital à la fois dans la conservation des produits culturels anciens et dans le transfert de cette culture de l'Orient vers l'Occident. Les califes abbassides encourageaient ce travail et ceci se manifesta avec la fondation en (829) de Bayt al-Hikma, ou Maison de Sagesse par Al-Ma'mûn, qui fut une véritable première académie de traduction d'œuvres grecques et syriaques, une bibliothèque et un observatoire d'astronomie. Les Omeyyades, avant les Abbassides, avaient eux aussi déjà incité les savants à traduire, en particulier à partir du grec, des livres de médecine, d'astronomie, de mathématiques et de philosophie ${ }^{28}$.

Dans ce contexte propice à la production des connaissances, la traduction a commencé à s'imposer non seulement comme l'un des outils de production du savoir scientifique mais aussi comme un moyen d'intercommunication entre les populations arabes et non arabes mais islamisées et qui maîtrisaient de nombreuses langues comme le grec, le syriaque, le persan, le turc ou le sindhi.

Sous l'Empire ottoman, la langue arabe a connu un certain recul. Mais elle connaîtra un renouveau grâce à la traduction à partir des langues européennes et au milieu du XIXe siècle, le Liban connaît un

\footnotetext{
${ }^{28}$ Cf. Taïeb BACCOUCHE, « La traduction dans le monde arabe », in Meta, Volume 45, Numéro 3, septembre 2000, pp. 395-399.
} 
mouvement de traduction important dans les domaines de la presse et de l'enseignement ${ }^{29}$.

Les Arabes n'étaient pas ainsi de simples passeurs qui ont transmis les différentes cultures anciennes (grecque, perse, hindoue) aux Européens, mais ils les ont enrichies, développées, affinées en les soumettant à leur propre pensée et à leur mode de raisonnement.

La langue arabe est donc comme nous l'avons précédemment signalé, une langue importante sur le marché national et international. Une entreprise pourrait attirer plus de clients et améliorer sa rentabilité non seulement en mettant en œuvre une stratégie d'affaires électroniques, en modifiant les prix ou les modalités de la facturation de ses produits, en surveillant ses concurrents sur le marché, en parrainant des activités communautaires ; mais aussi en sachant comment adapter un produit à un marché cible; ou autrement dit, en le localisant pour qu'il porte l'empreinte de ce marché. Et puisque la langue est porteuse d'identité, de valeurs, d'histoire et de sens, celle-ci parvient de la sorte à souder la cohésion sociale et favoriser le développement d'un sentiment d'appartenance à la collectivité. Localiser un produit, signifierait, donc, de prime abord, savoir adapter sa langue, son contenu et son apparence au public cible.

\section{La localisation et la traduction : convergences et divergences :}

Tout ce qui précède nous mène à deux questions cruciales qui continuent à hanter les esprits de tous ceux qui s'intéressent au domaine de la traduction et qui voient, dans tout ce qui est nouveau une sorte de menace: La localisation se situe-t-elle sur un même pied d'égalité avec la traduction ou la dépasse-t-elle ? Localisation et traduction sont-elles deux opérations distinctes ou deux pièces complémentaires dans un même puzzle?

A la première question, nous pourrons répondre par "non". La localisation ne se situe pas sur un même pied d'égalité avec la localisation, mais la dépasse pour plusieurs raisons. Tout d'abord, parce que la localisation intègre une dimension culturelle inhérente surtout à l'internationalisation du commerce mondial. En d'autres termes: "La localisation, processus d'adaptation d'un produit ou d'un service auprès d'une région spécifique », ajoute "un aspect inédit à la traduction. C'est un processus dans lequel la culture de chaque pays cible prend toute son importance et sa dimension ${ }^{30}$. Ainsi, en localisation plus qu'en traduction, le lien

${ }^{29}$ Cf. Ibid. Loc.cit

30 «Localisation et traduction : quelles différences ? , in https://culturesconnection.com/fr/localisation-traduction-differences/ Dernière consultation le 8 juillet 2019.

\section{3}


avec les mots est ingénieux : "le traducteur, habitué aux coutumes et traditions d'une langue, est capable d'en percevoir les nuances et sa traduction finale n'en sera que de meilleure qualité. Par conséquent, le mécanisme de localisation suppose une compréhension profonde des dispositifs qui dominent les deux cultures sources et cibles » ${ }^{31}$.

Aussi, la localisation, diffère de la traduction sur le plan du transfert linguistique, c'est-à-dire le passage d'une langue à une autre. En effet, la spécificité de la localisation par rapport à la traduction réside dans le fait qu'il n'y a pas de « texte de départ » au sens où on l'entend traditionnellement. Le contenu textuel à localiser, le document Web source, n'est destiné à aucun public en particulier et n'est connu que des agences de localisation. Le contenu textuel est d'abord internationalisé, c'est-àdire rédigé en anglais, exempt de termes, de connotation et de dénotations culturelles, dans le but de faciliter son adaptation à l'échelle planétaire. Il est ensuite localisé pour faire référence aux éléments spécifiques à une communauté linguistique donnée au sein d'une région ; c'est-à-dire pour être conforme au droit, à la religion ou encore à d'autres aspects culturels de la clientèle visée. En ce sens, «la localisation est une version plus aboutie de la traduction parce que plus complète $»^{32}$ et plus fouillée .

Cependant, quelles que soient ces divergences, la réponse à la deuxième question, montrera que la localisation et la traduction ne sont pas deux opérations totalement distinctes mais plutôt complémentaires. En effet, la traduction reste au cœur du processus de localisation. Celle-ci est une étape indispensable qui contribue à donner l'impression que le site web, le logiciel ou la documentation a été créé directement pour le marché cible. En plus, ce qui accentue cette complémentarité, c'est que dans le processus de la localisation, le localisateur porte les deux étiquettes de traducteur-localisateur. Autrement dit, le localisateur est capable non seulement de traduire mais aussi «d'extraire le contenu à localiser et de le réintégrer après sa localisation $\gg$. Sinon, il demeure uniquement un "localisateur-linguistique" qui se fait aider toujours par un 'localisateur de plein exercice'.

\section{Le processus et les outils de localisation :}

Comme nous venons de le souligner, la traduction constitue un des maillons dans une seule et même chaine, celle de la localisation. Ainsi, tout projet de localisation d'un produit, ne se limite pas à la seule tâche de traduction, mais se compose d'un

\footnotetext{
${ }^{31}$ Ibid., Loc. cit.

${ }^{32}$ Ibid., Loc. cit.

${ }^{33}$ Daniel GOUADEC, « Le bagage spécifique du localiseur/localisateur : Le vrai nouveau profil requis », in Meta Volume 48, Issue 4, Décembre 2003, p.126.
} 
long processus comportant d'autres opérations d'égale importance, lesquelles sont, selon ESSELINK ${ }^{34}$, SCHÄLER $^{35}$ et l'entreprise ARABIZE ${ }^{36}$ : la phase de prévente, la réunion de lancement, l'analyse, la traduction, la partie technique, le contrôle de qualité et livraison et enfin, la clôture du projet.

«La phase de prévente » commence avant qu'un projet ne soit attribué à une agence de localisation. Dans cette phase, des demandes de devis ou des demandes de propositions sont généralement envoyées par le client à plusieurs agences afin d'obtenir la meilleure offre. Ces demandes sont généralement accompagnées d'un CD comportant le contenu source afin de permettre aux agences de localisation de créer un devis et une proposition de projet. Les différentes agences évaluent donc le contenu source et fournissent un devis ainsi qu'une répartition de toutes les activités du projet.

Une fois le projet attribué à une de ces agences, les éditeurs de logiciels organisent « une réunion de lancement » avec les traducteurs, les ingénieurs de localisation et les gestionnaires de projet afin de leur donner un aperçu sur le projet et leur expliquer les procédures et démarches qu'ils doivent suivre dans ce projet.

Après ces deux premières phases, vient « la phase d'analyse » du projet. Dans cette phase, le contenu source, est évalué et analysé par les spécialistes d'après la version anglaise du produit, les versions précédemment traduites du même produit, et les glossaires ou les mémoires de traduction comportant la terminologie propre au projet. Notons que les grandes agences de localisation possèdent généralement une équipe d'évaluation de projet dont le rôle principal est la stricte analyse des différents aspects d'un nouveau projet de localisation, l'identification des éléments qui pourraient lui être problématiques, la sélection des outils qui lui seront indispensables et la fourniture aux gestionnaires du projet de toutes les informations nécessaires pour créer un calendrier au projet et un budget.

La phase d'analyse constitue la base de la phase suivante: celle de «la préparation ». Dans celle-ci, les chefs de projet, les techniciens et les coordinateurs linguistiques préparent un plan (qui détermine les tâches, les étapes et les détails budgétaires) et un kit de localisation contenant tout le matériel nécessaire pour la localisation (les contenus sources, les outils à utiliser, les directives de traduction et

\footnotetext{
${ }^{34}$ Cf. Bert ESSELINK, Op.cit., p.17-23. Notre traduction.

${ }^{35}$ Cf. Reinhard SCHÄLER, Localization dans Mona BAKER et Gabriela SALDANHA, Routledge encyclopedia of translation studies, 2ème edition, Abingdon, Routledge, 2009, p.159. Notre traduction

${ }^{36}$ Ces étapes m'ont été expliquées lors de mon stage « Formation de formateurs en localisation », en 2015 au sein de l'entreprise Arabize.
}

515 
de localisation, le système de repérage des fautes, les instructions pour la remise du produit), qui seront fournis à tous les membres de l'équipe. De plus, la phase de préparation peut comporter également la création d'un glossaire multilingue contenant tous les termes liés au produit en question et indiquant ceux qu'il faut garder dans la langue originale.

Quant à «la phase de traduction", elle ne diffère pas beaucoup de celle d'une traduction ordinaire. Évidemment, cette traduction localisée ne se limitera pas uniquement au contenu textuel, mais comportera aussi, les menus, les boîtes de dialogues, les messages, l'aide en ligne, les images contenant du texte dans le cas d'un logiciel ou d'un site web.

Après cette phase, il faut réviser minutieusement la traduction en comparant les deux textes source et cible avant de remettre le projet aux informaticiens. Dans cette phase technique, les informaticiens commencent par restructurer l'interface, selon le besoin, et ce en élargissant par exemple les espaces, les fenêtres ou les touches trop petites par rapport au texte traduit en langue cible. Dans la localisation des logiciels, ils sont également chargés de donner des raccourcis clavier adéquats (par rapport aux commandes traduites).

Lorsque toutes ces phases sont accomplies, le travail sera prêt pour être soumis à un «contrôle de qualité », qui prévoit plusieurs tests. D'habitude, le test linguistique est fait par les linguistes. Il y a également un test des fonctionnalités. Après cela, tout le matériel est envoyé à l'éditeur qui doit l'approuver. Le projet est donc ainsi presque finalisé.

Reste une dernière phase, celle de «la clôture du projet». En fait, lors de cette phase, de nombreux éditeurs organisent une réunion de vérification post-mortem avec l'agence de localisation après la fin d'un projet. Lors de ces réunions, on évalue le processus du projet finalisé, la qualité technique et linguistique du travail remis ; on suggère les points à améliorer, s'il y en a, pour une meilleure coopération future.

Pour mener à bien ce long processus de localisation, différents outils entrent en jeu, à savoir des outils de gestion, utilisés pour dresser un plan du projet, gérer le processus de localisation dans sa totalité et favoriser l'interaction entre les différents acteurs; des outils de traduction comme les logiciels de traduction automatique (TA), les mémoires de traduction (MT), les bases de données terminologiques, des outils de vérification qui assurent la correction de la traduction, et des outils techniques de mise en page soit pour les logiciels, soit pour les sites web. Ces quatre grandes catégories d'outils nécessitent une assimilation profonde et une expérience prolongée afin de pouvoir les maîtriser et les manipuler correctement.

\section{Les différents types de localisation les plus fréquents :}


Il y a plusieurs types de localisation qui, tous, sont soumis au même processus déjà évoqué et où l'on utilise les mêmes outils. Parmi ces types, nous pourrons citer : la localisation des logiciels, la localisation des sites web, la localisation des publicités, la localisation des produits, la localisation de la documentation technique et la localisation des multimédias.

\subsection{La localisation des logiciels :}

La localisation logicielle porte sur la traduction et l'adaptation complètes des logiciels informatiques et de leurs manuels, comme les logiciels de traitement de texte tels que Word. C'est un processus qui consiste aussi à adapter l'interface utilisateur du produit, les fenêtres, les menus, les boutons, les boîtes de dialogue, les systèmes d'aide en ligne, les applications mobiles ou web, le redimensionnement d'interfaces ... etc. ${ }^{37}$

La localisation de ces logiciels passe par plusieurs étapes dont les plus importantes sont les suivantes: extraire le texte à traduire, rechercher les équivalences terminologiques, analyser les interfaces à adapter, constituer des glossaires spécialisés, coordonner avec les manuels techniques, affecter les touches d'accès rapide, corriger et intégrer la langue, réviser les fonctionnalités, tester la facilité d'utilisation du logiciel, essayer l'exploration du logiciel dans des conditions réelles et produire le logiciel de façon définitive accompagné de toute la documentation nécessaire à l'utilisateur.

Nous avons utilisé comme exemple, deux versions de la même page du logiciel Microsoft Word. La première est tirée de la version française (Figure 5) et la seconde de celle de l'Égypte (Figure 6). Nous pouvons constater que les deux versions sont identiques puisqu'elles partagent la même mise en page, les mêmes boutons présentés dans le même ordre. Une seule différence pourrait être repérée, celle de la direction de la page qui change d'après la langue : de gauche à droite pour la langue française et de droite à gauche, pour la langue arabe.

${ }^{37} \mathrm{Cf}$. http://www.sdltrados.com/fr/solutions/software-localization/ Dernière consultation le 05/12/2018. 

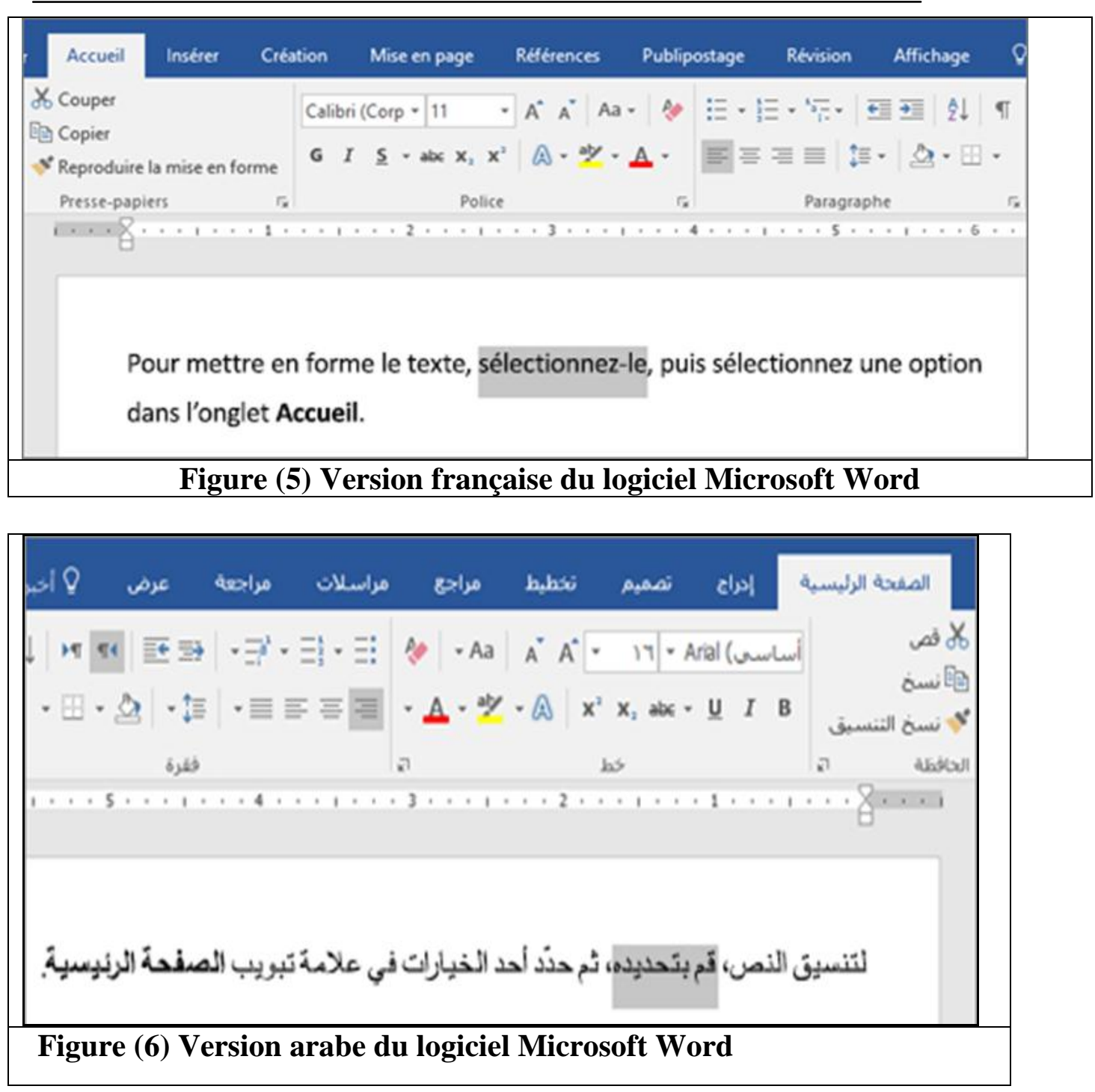

○

\subsection{La localisation des sites Web :}

Le second type de localisation, très en vogue comme le précédent, est celui de la localisation des sites Web qui comprend la traduction et la localisation du contenu informationnel et textuel - de façon à la fois précise et nuancée dans la langue ou le dialecte de la société ou du pays cible- des images, des icônes et des formats dans une culture donnée. Aussi allons-nous trouver que presque tous les composants du site doivent être modifiés et adaptés à la langue et à la culture de la société d'accueil. Nous pourrons mentionner à titre d'exemple :

-Le format des dates et calendriers : 
Le format des dates est un élément très important dans la localisation; puisque celui-ci n'est pas le même partout. En effet, il y a deux formats pour l'affichage de la date : «little endian» (jour/mois/année), et le format «middle-endian» (mois/jour/année) ${ }^{38}$ comme l'exemple de l'heure de l'arrivée à l'hôtel, sur le site Booking.com ${ }^{39}$ Nous remarquons que la version anglaise ( Figure 7) opte pour le « middle-endian » alors que les deux sites français (Figure 8) et arabe ( Figure 9) utilisent le format de date « little endian » .

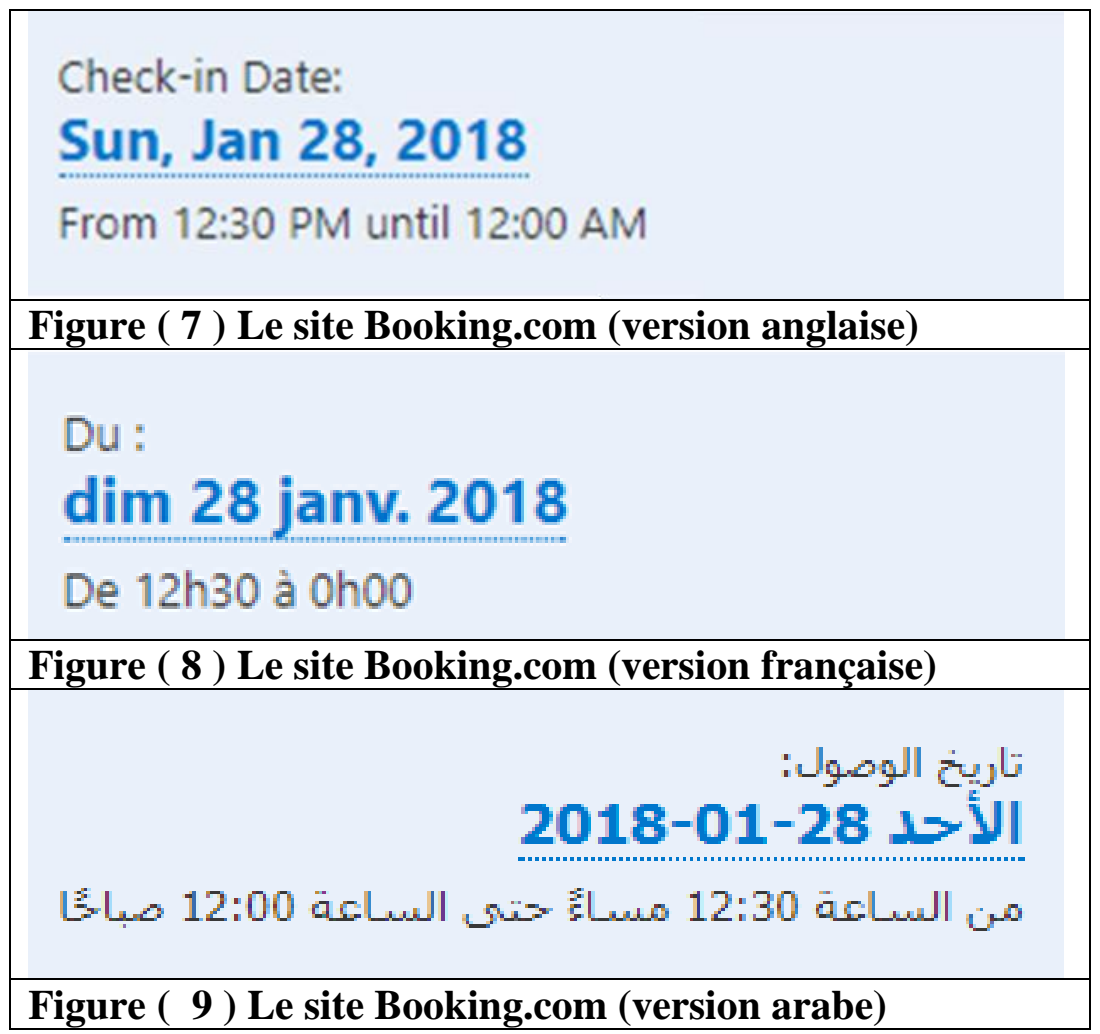

Dans la localisation, il faut également faire attention au type de calendrier utilisé ${ }^{40}$ dans le pays cible, surtout s'il est question de pays arabophones, puisque le calendrier adopté en Egypte par exemple est différent de celui adopté dans les pays du Levant ou les pays du Golfe. En fait, en arabe, un pays comme l'Egypte suit par

\footnotetext{
${ }^{38}$ Pour plus d'informations, consultez le lien suivant : https://frenchmorning.com/pourquoi-lesamericains-mettent-le-mois-en-tete-de-la-date/. Dernière consultation le 19 janvier 2018.

${ }^{39}$ Booking.com, créé en 1996, est un site néerlandais de réservation d'hébergement en ligne.

${ }^{40} \mathrm{https} / / / \mathrm{cris} 23 . \mathrm{fr} / \mathrm{calendriers.htm}$

Dernière consultation le 09 janvier 2018.
}

519 
exemple le calendrier grégorien appelé aussi calendrier solaire occidental ( يناير) (فبراير، مارس،... ou (Janvier, février, mars,...) et musulman appelé aussi calendrier lunaire (محرم، صفر ، ربيع الأول) (Al-Mouharram, Safar, Rabi^ al-'awwal, Rabi^ al'akhir, Joumada 1-'oula, Joumada 1-'akhirah, Rajab, Cha^ban, Ramadan, Chawwal, Dhou 1-qa^dah, Dhou 1-hijjah) ${ }^{41}$, les pays du Levant suivent à la fois le calendrier grégorien et babylonien connu sous le nom de calendrier solaire oriental (كانون الثناني، شباط، آذار) musulman.

Ainsi, dans la version française du site de la compagnie Middle East Airlines (MEA), les dates des différents vols sont affichées selon le calendrier solaire occidental; sur le site libanais de la même compagnie, les dates des vols sont affichées selon le calendrier babylonien, comme le montre les deux figures (10 et $11)$.

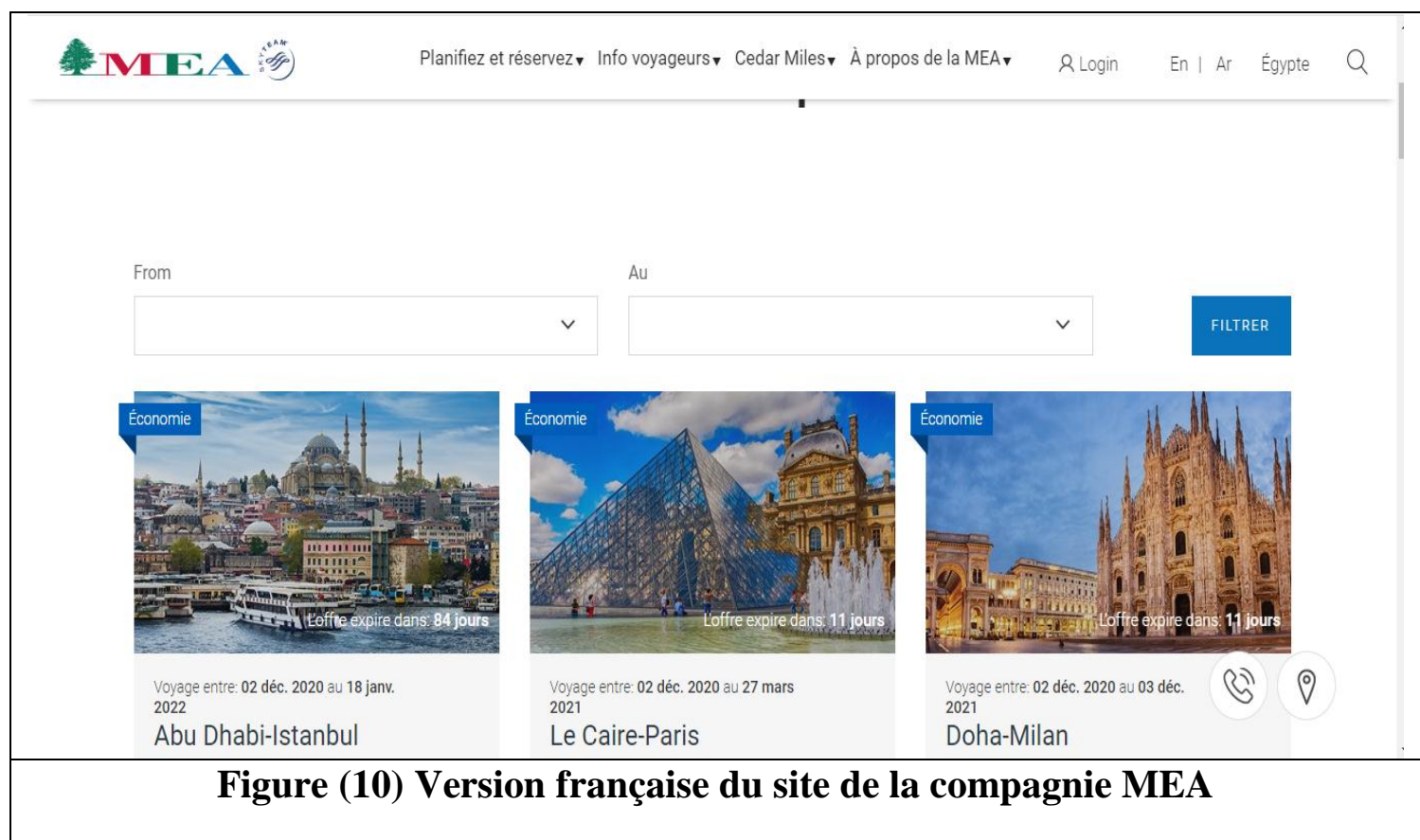

\footnotetext{
${ }^{41}$ https://www.apbif.fr/noms-des-mois-lunaires/ Dernière consultation le 09 janvier 2018.

${ }^{42}$ Pour plus d'informations, consultez le lien suivant : https://tresoreducatif.com/les-mois-de-lanneecalendrier-lunaire-et-solaire-oriental-et-occidental/, Dernière consultation le 09 janvier 2018.'
} 
-Le format des heures, horaires de travail et cordonnées ${ }^{43}$ :

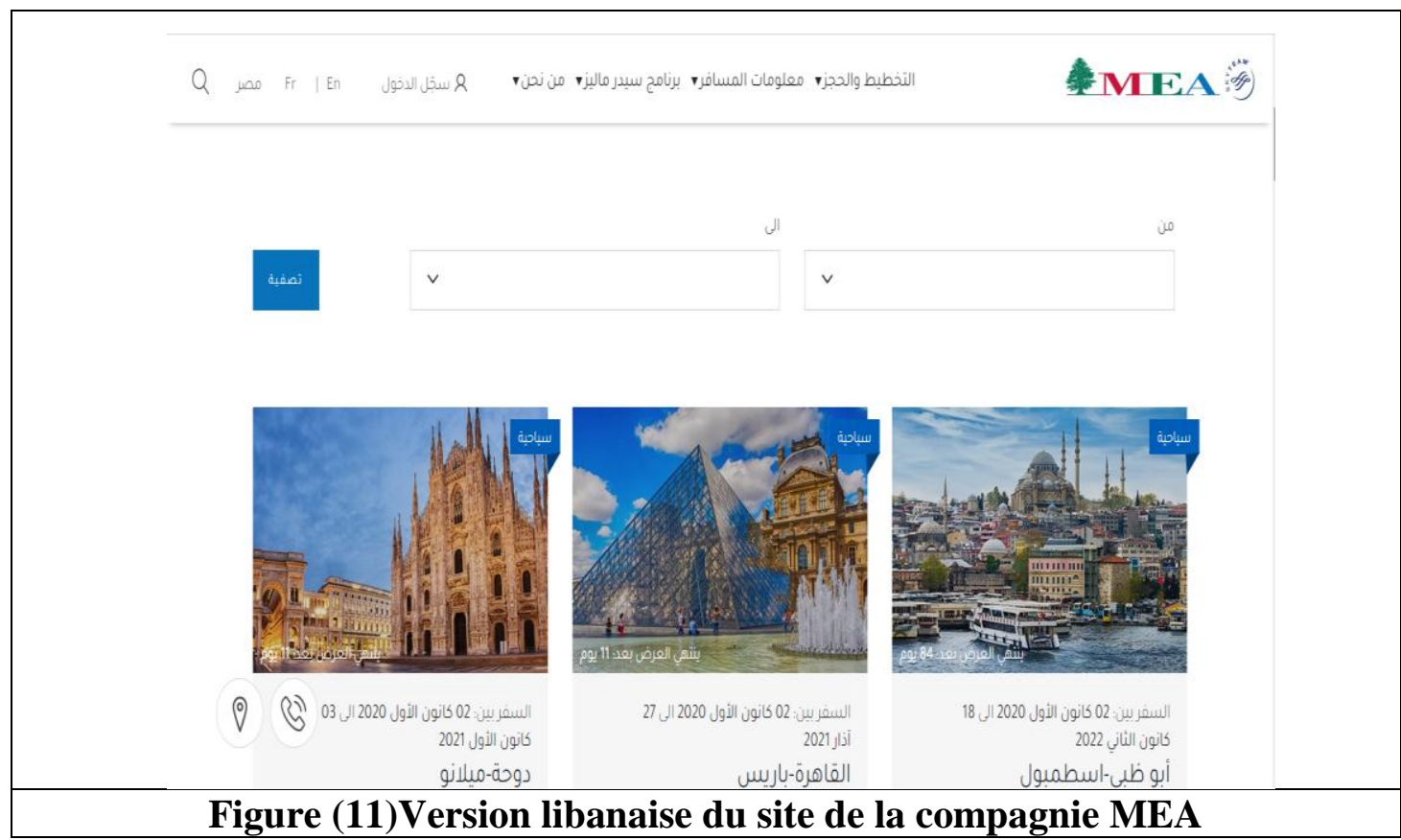

Les formats des heures et les horaires de travail doivent aussi être localisés pour correspondre à la société cible. En fait, il y a deux formats d'affichage d'heures : le format d'affichage 12 heures (AM/PM) et le format d'affichage 24 heures. Les horaires de travail et les jours de congés diffèrent d'un pays à l'autre : les pays arabes, à l'exception de ceux du Levant et du Maghreb, par exemple adoptent le format des 12 heures dans l'affichage de leurs horaires et considèrent le samedi comme le début de la semaine. Alors que les pays européens adoptent le format des 24 heures et considèrent le lundi comme le début de la semaine.

Ainsi, le site officiel de la compagnie Egyptair ${ }^{44}$, est traduit littéralement en toutes les langues présentes dans la rubrique «paramètres ». Le contenu du site ainsi que

\footnotetext{
${ }^{43}$ https://support.minitab.com/fr-fr/minitab/18/help-and-how-to/manipulate-data-in-worksheetscolumns-and-rows/supporting-topics/date-time-data-and-formats/defining-custom-date-time-displayformats/

Dernière consultation le 02/04/2020

${ }^{44}$ https://www.egyptair.com/en/Pages/HomePage.aspx

Dernière consultation le 02/06/2020
}

521 
les rubriques sont identiquement les mêmes dans toutes les langues. Au niveau de la localisation, et en examinant le site d'Egyptair ( Figure 12) présent en Egypte ${ }^{45}$ et celui présent en France ${ }^{46}$ ( Figure 13), nous allons remarquer que la localisation du site égyptien porte surtout, sur les horaires de travail, les formats des heures et les cordonnées qui diffèrent dans les deux versions. Nous allons trouver donc qu'en Egypte, Egyptair ouvre ses portes tous les jours de la semaine de 9 à 5 heures alors qu'en France, elle n'ouvre ses portes que du lundi au vendredi de 9.30 à 17 heures. Le weekend, samedi, dimanche est donc maintenu en France. Les adresses (codes postaux, numéro des routes, codes des provinces, etc.), ainsi que les numéros de téléphone ont été également localisés sans négliger l'ajout des codes régionaux de téléphone. Le localisateur, conscient de la différence des horaires de travail ainsi que du format des heures et des cordonnées entre les deux pays, l'Egypte et la France, a tenu à les respecter dans sa localisation de la version égyptienne pour qu'elle soit adaptée au public français.

${ }^{45}$ https://www.egyptair.com/ar/contact-us/Pages/DomesticOffices.aspx Dernière consultation le 02/06/2020

${ }^{46}$ https://www.egyptair.com/fr/contact-us/Pages/WorldWideOffices.aspx Dernière consultation le 02/06/2020 

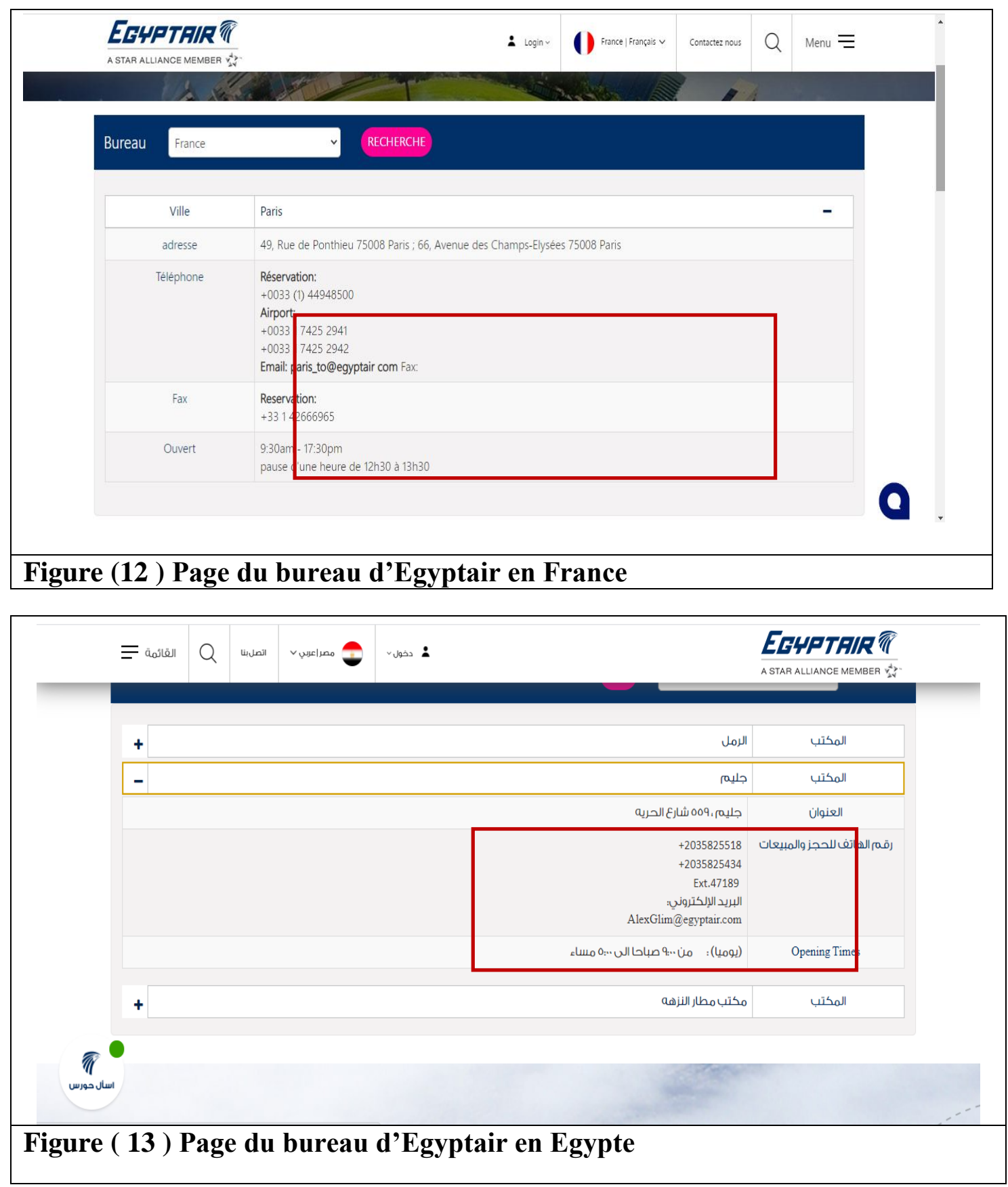

-Les devises et les modes de payement

Pour les devises, le localisateur doit également tenir compte de la différence de devises entre les différents pays. Une devise étrangère donne une impression de distance et ne rassure pas le consommateur. Cependant, le fait de convertir la devise pour qu'elle corresponde au marché cible réduit cet écart interculturel existant entre deux communautés différentes. Ainsi, sur le site de vêtements prêt-à-porter H\&M,

\section{3}


localisé pour l'Egypte ${ }^{47}$, tous les prix sont affichés en Livre Egyptienne ( Figure 14) et non en Euro pour que l'utilisateur puisse se retrouver facilement sans avoir à convertir vers la devise de son pays. Alors que sur le site français ${ }^{48}$, tous les prix sont affichés en euros (Figure 15).

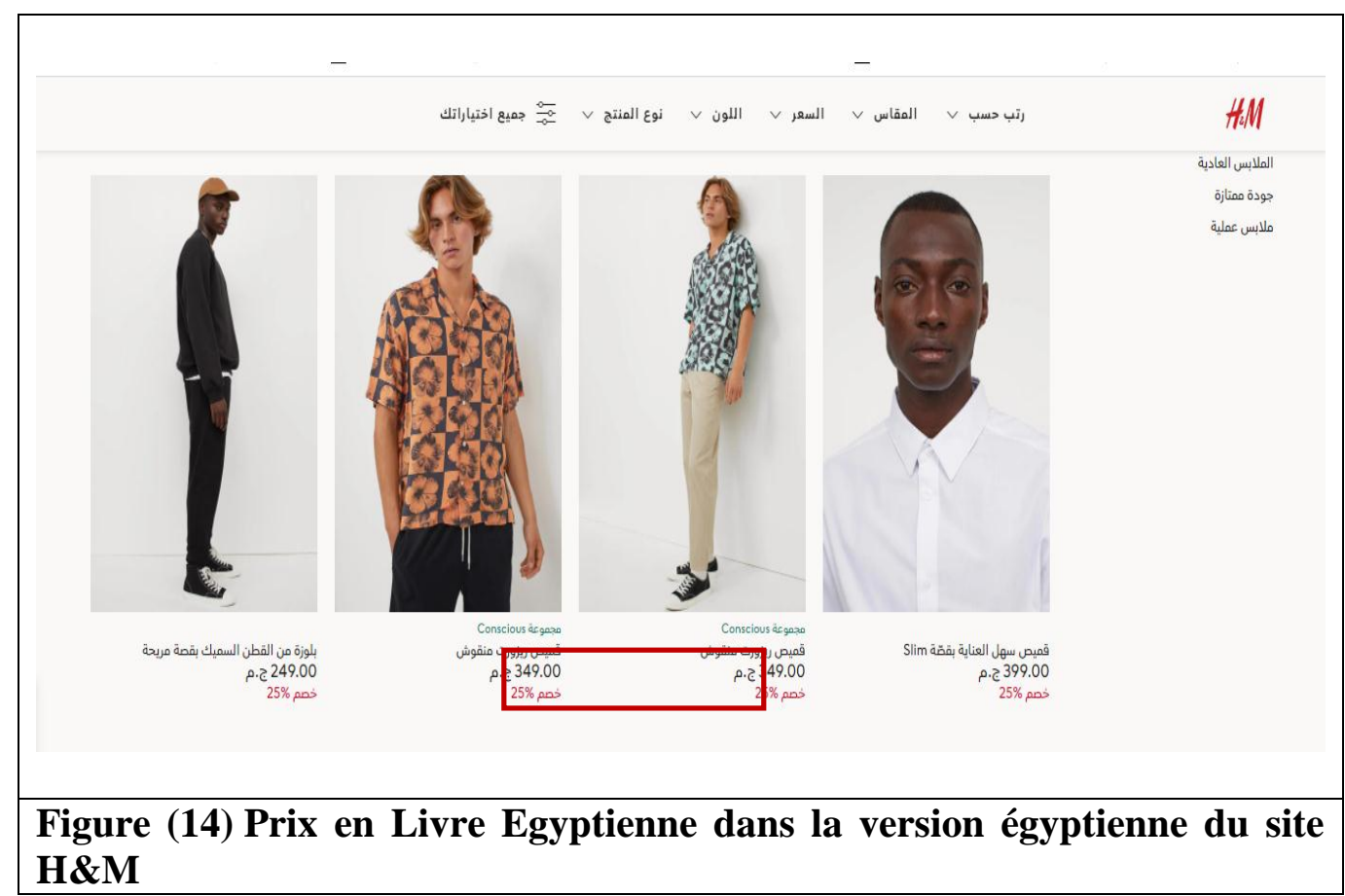

${ }^{47}$ https://eg.hm.com/ar/shop-men/shop-product/shirts/

Dernière consultation le 09/09/2019

${ }^{48} \mathrm{https}: / /$ eg.hm.com/en/?utm_source=partnerize \&utm_medium=affiliate\&utm_campaign=110111037 $15 \& u t m \_$content $=0 \&$ utm_term $=$

Dernière consultation le 09/09//2019 


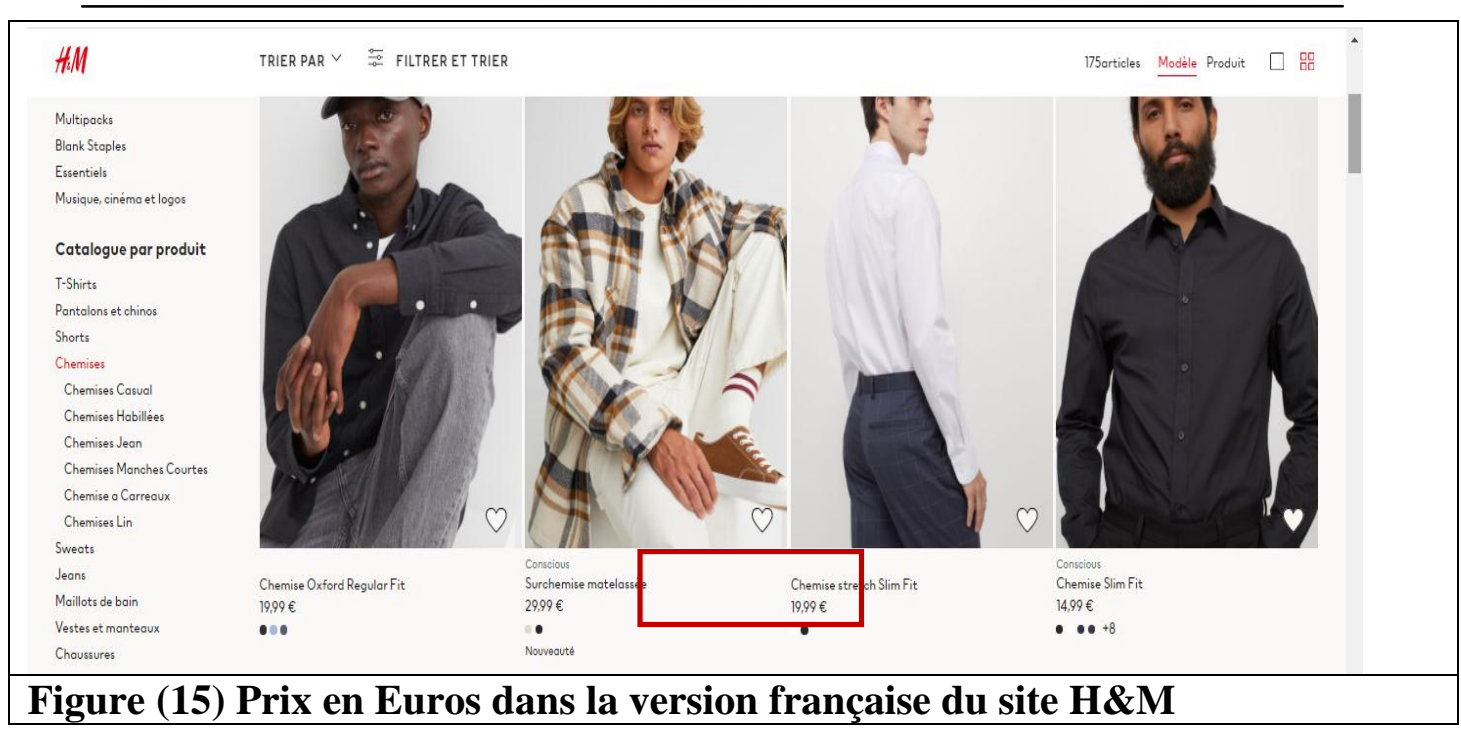

\section{-La direction de la page :}

La direction de la page diffère selon la langue. Ainsi, comme nous l'avions précédemment évoqué, en français, la direction de la page est de gauche à droite, tandis qu'en arabe, de droite à gauche. Ce concept touche non seulement la direction des mots mais également celle des images, des organigrammes, et des tableaux comme le montre les figures ci-dessous : la (Figure16) montre la différence entre la direction de l'avion dans la version française de la compagnie Egyptair ${ }^{49}$ et celle de l'avion dans la version arabe de la même compagnie ${ }^{50}$. Quant à la (Figure 17), celle-ci montre le changement dans la disposition des tableaux dans les deux versions, arabe et française, du même manuel de fax 2840 de Brother $^{51}$.

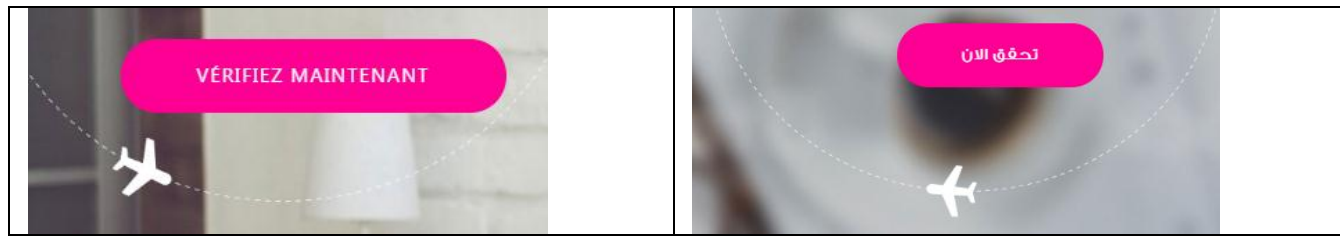

\section{${ }^{49}$ https://www.egyptair.com/fr/contact-us/Pages/WorldWideOffices.aspx Dernière consultation le 02/06/2020 \\ ${ }^{50} \mathrm{https} / / /$ www.egyptair.com/ar/contact-us/Pages/DomesticOffices.aspx} Dernière consultation le 02/06/2020

${ }^{51}$ Brother, créée au Japon en 1908, est une marque spécialiste des périphériques d'impressions (imprimantes et multifonctions).

525 
Figure (16) Comparaison de deux versions de la même page du site d'Egyptair.

\begin{tabular}{|c|c|c|c|}
\hline Bacs & Nom & الاسم & اللازج و الوحدة الاختيارية \\
\hline Bac à papier standard & Bac1 & الدرج1 & درج الورق القيانسي \\
\hline $\begin{array}{l}\text { Dispositif d'alimentation } \\
\text { manuelle }\end{array}$ & Manuelle & بدوي & فتحة التُغذية الليدوية \\
\hline
\end{tabular}

Figure (17)

Comparaison de deux versions du même tableau du manuel du fax 2840 de Brother $^{52}$.

\section{-L'adresse URL:}

Les adresses URL doivent être aussi localisés. En fait, adapter les URL des pages web au pays cible ou à la langue cible aura un impact positif sur le référencement du site. Ainsi, dans l'adresse URL de la version française du site de l'ONU ${ }^{\mathbf{5 3}}$ (Figure 18) le lien est le suivant: https://www.un.org/fr , et dans la version arabe du site de l'ONU, (Figure 19) le lien est le suivant: https://www.un.org/ar . Une seule différence pourrait être décelée, celle de la présence de la langue du site dans l'adresse des deux sites : « $\mathrm{Fr}$ » pour la langue française et « $\mathrm{Ar}$ » pour la langue arabe. Cependant, cette différence, bien que minime, s'avère indispensable dans la localisation du site. Il est donc essentiel que chacune des pages de langues différentes ou de pays différents soit affichée avec sa propre URL accessible, afin qu'elle puisse être suivie, indexée et positionnée par Google ou n'importe quel autre moteur de recherche.

\footnotetext{
52 https://www.brother.fr/services-et-supports/fax-2840/manuals

Dernière consultation le $09 / 07 / 2020$

${ }^{53}$ ONU (Organisation des Nations unies), fondée en 1945, est une organisation internationale regroupant, à quelques exceptions près, tous les États de la Terre. Ses objectifs sont de faciliter la coopération dans le droit international, la sécurité internationale, le développement économique, le progrès social, les Droits de l'homme et la réalisation à terme de la paix mondiale.
} 
-Les lettres majuscules:

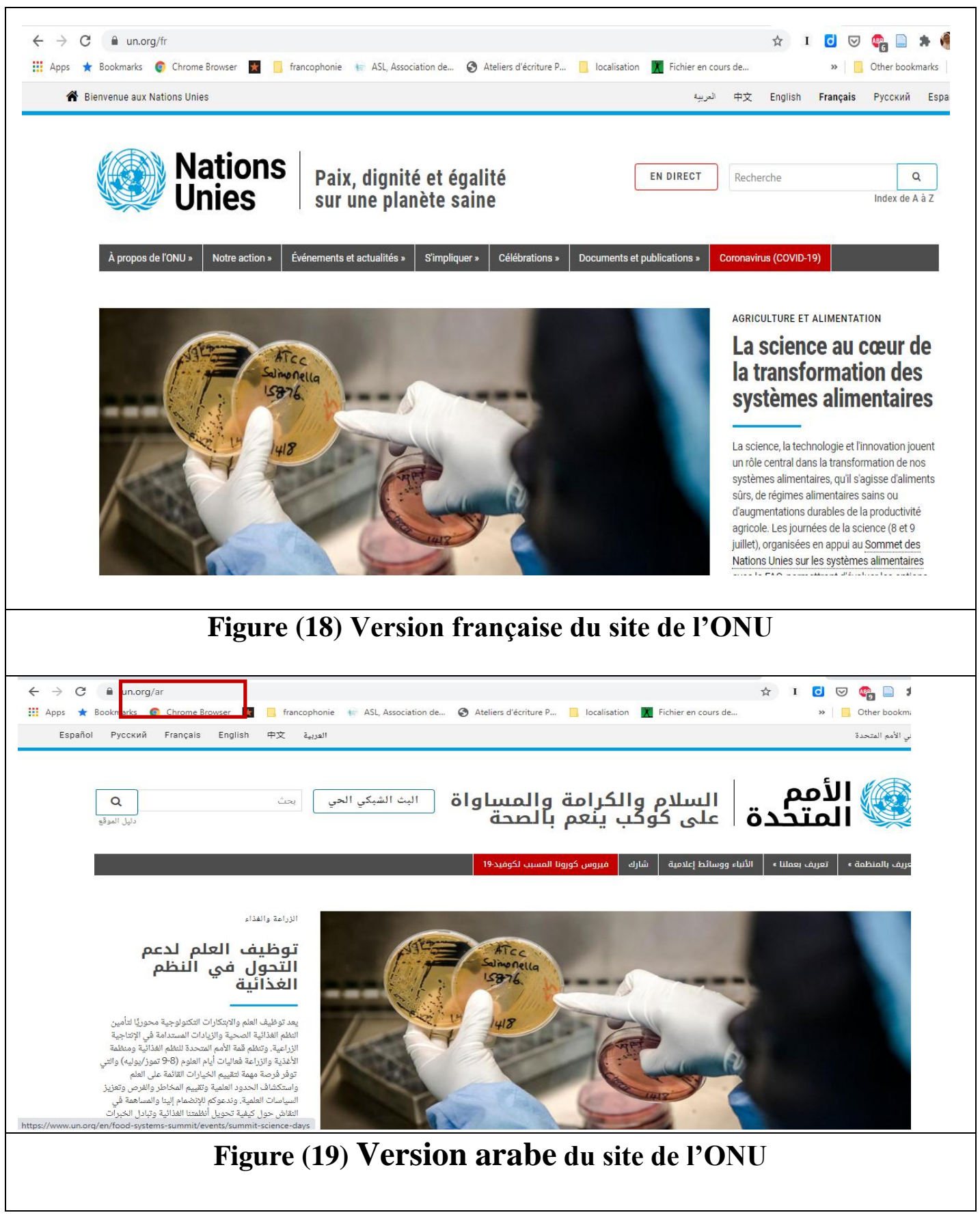

Puisque les lettres majuscules n'existent pas en arabe, les localisateurs ont alors recours à des solutions alternatives pour souligner certaines consignes ou 527 
fonctionnalités. Ainsi, les mots qui commencent par des lettres majuscules sont remplacés dans la langue cible - s'il s'agit de la langue arabe - par des italiques, ou des caractères gras, ou sont mis entre guillemets. Nous pouvons remarquer cela dans la version française du support Microsoft $^{54}$ (Figure 20) où les mots «Disposition » et «Marges » sont écrits en gras et commencent par des majuscules tandis que dans la version arabe (Figure 21) du même support ${ }^{55}$, les mots arabes

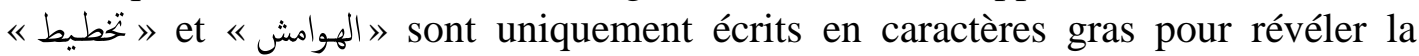
même importance et parce qu'il n'y a pas de lettres majuscules en arabe.

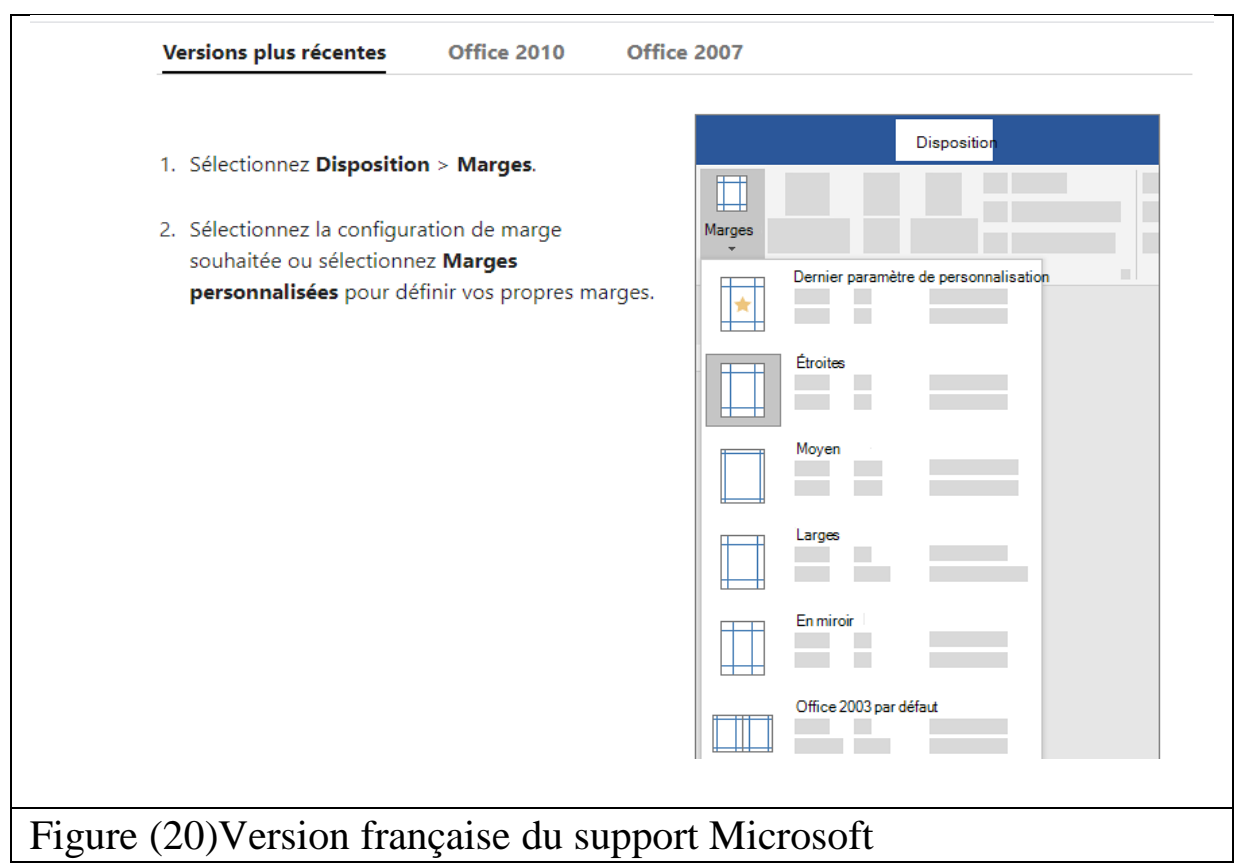

\footnotetext{
${ }^{54}$ https://support.microsoft.com/fr-fr/office/changer-les-marges-da21a474-99d8-4e54-b12da8a14ea7ce02?wt.mc id=fsn_word lay out pages Dernière consultation le 03/08/2020

${ }^{55} \mathrm{https}$ ://support.microsoft.com/ar-sa/office/\%D8\%AA\%D8\%BA\%D9\%8A\%D9\%8A\%D8\%B1\%D8\%A7\%D9\%84\%D9\%87\%D9\%88\%D8\%A7\%D9\%85\%D8\%B4-da21a474-99d8-4e54-b12da8a14ea7ce02?wt.mc id=fsn_word_lay_out_pages Dernière consultation le 03/08/2020
} 


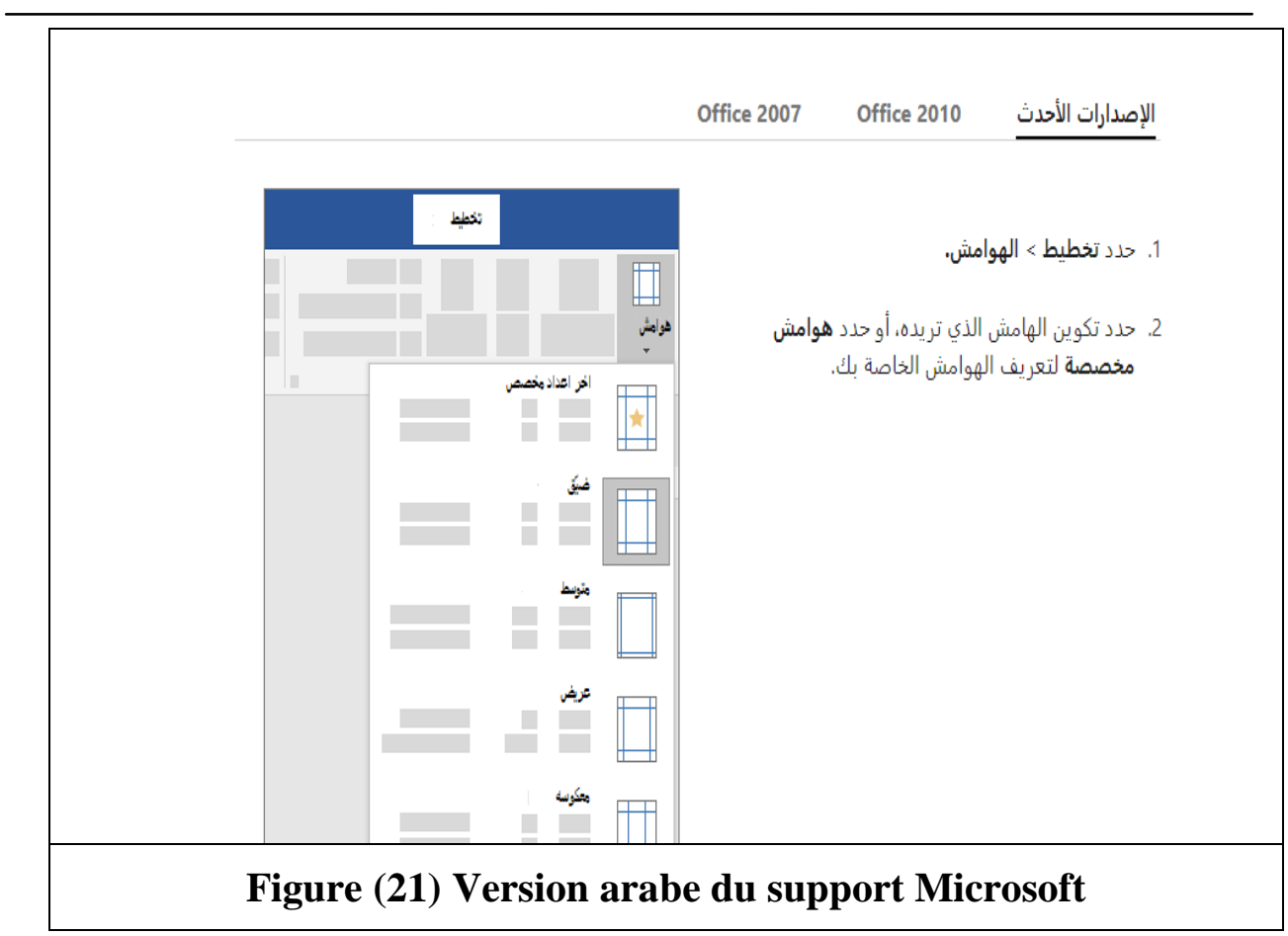

\section{-Les pages HTML :}

En localisant un site, il faut aussi faire attention au langage HTML ${ }^{56}$ qui gère les différentes pages d'un site car ce langage contient des balises et donc n'importe quel changement pourrait endommager la structure de la page et compromettre ainsi son fonctionnement comme le montre l'exemple de la (Figure 22). Dans cet exemple, lorsque la page sera localisée en arabe, il faut faire attention au langage de programmation de chaque partie dans la page. Ainsi, pour le titre de la page par exemple, la seule partie traduisible du code sera le titre «Bienvenue ! » puisque tout élément HTML se compose d'une balise de début et de fin, qui dans ce cas est « title» en plus de la balise qui détermine la couleur.

\footnotetext{
${ }^{56}$ HTML (HyperText Markup Language) est le langage de balisage conçu pour représenter les pages web. L'HTML est ce qui permet à un créateur de sites Web de gérer la manière dont le contenu de ses pages Web va s'afficher sur un écran, via le navigateur. Il repose sur un système de balises permettant de titrer, sous-titrer, mettre en gras, etc., du texte et d'introduire des éléments interactifs comme des images, des liens, des vidéos.

https://www.journaldunet.fr/web-tech/dictionnaire-du-webmastering/1203255-html-hypertext-markup-

langage-definition-traduction/

Dernière consultation le 07 juillet 2019
}

529 


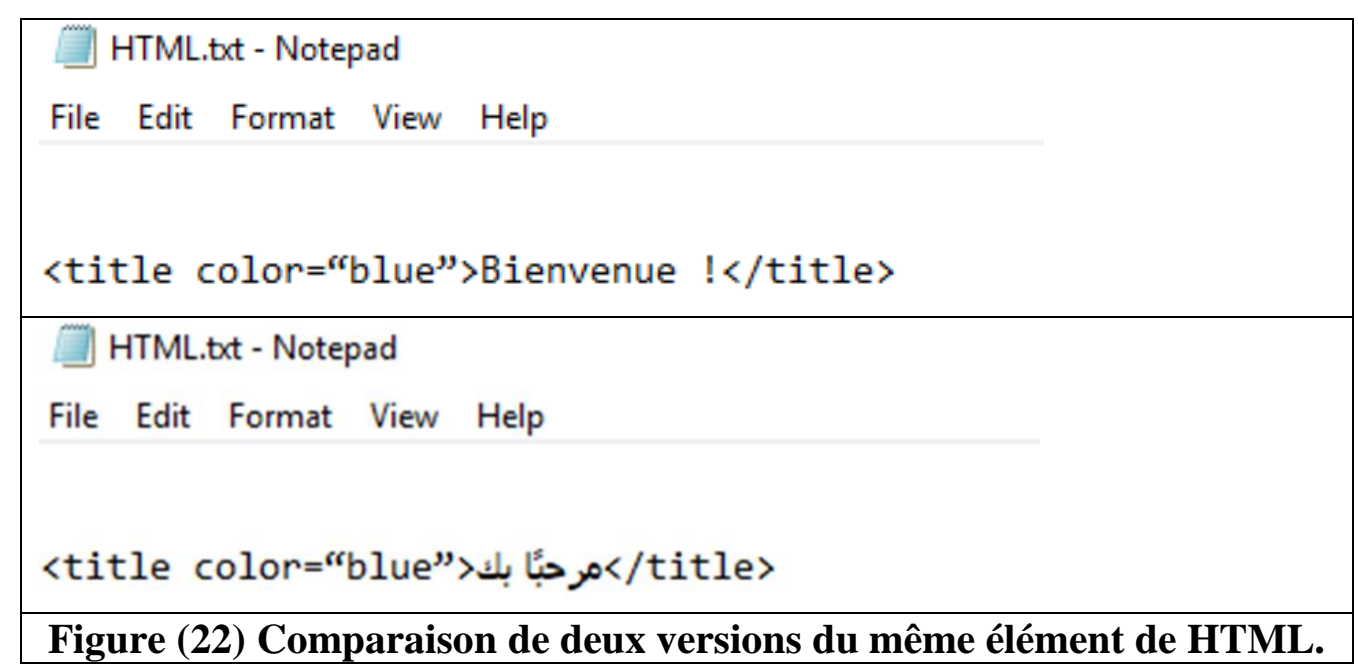

\section{-Image et contenu textuel :}

Adapter le contenu textuel et les images en fonction du marché ciblé s'avère très important afin de présenter aux internautes de ce marché ciblé un contenu adapté à leurs habitudes comportementales, us et coutumes.

De ce fait, les images insérées dans un produit localisé (site, affiche, campagne publicitaire, etc) doivent être dépourvues de tout élément non convenable à la culture cible. Citons l'exemple du logo de Starbucks lors de l'arrivée de la marque sur le marché saoudien en septembre 2000. A l'époque, la marque n'avait pas réalisé un grand succès et avait suivi le conseil du groupe de distribution koweitien avec qui elle s'est alliée au Moyen-Orient. Pour conclure son affaire avec l'Arabie saoudite, celui-ci lui avait conseillé d'utiliser un logo modifié, sans la sirène. Peter Maslen, le président de Starbucks Coffee International justifiait ce choix en janvier 2002 dans le Washington Post en disant qu'en tant que compagnie qui entre sur de nombreux marchés internationaux, ils sont très sensibles et grandement respectueux aux traditions religieuses locales, aux normes sociales et aux lois. Il ajouta également que l'enseigne ne souhaitait pas imposer ses valeurs à ses partenaires. Néanmoins quelques mois plus tard, en avril 2002, la police religieuse saoudienne, avait finalement accepté la présence de la sirène sur le logo de la chaîne de cafés. La sirène a donc rapidement refait son apparition sur les enseignes et les tasses Starbucks d'Arabie saoudite ${ }^{57}$ (Figure 23) .

\footnotetext{
${ }^{57}$ Cf. https://www.liberation.fr/desintox/2015/10/20/non-l-arabie-saoudite-n-a-pas-noye-la-sirenestarbucks_1407553/

Dernière consultation le 20/10/2020
} 


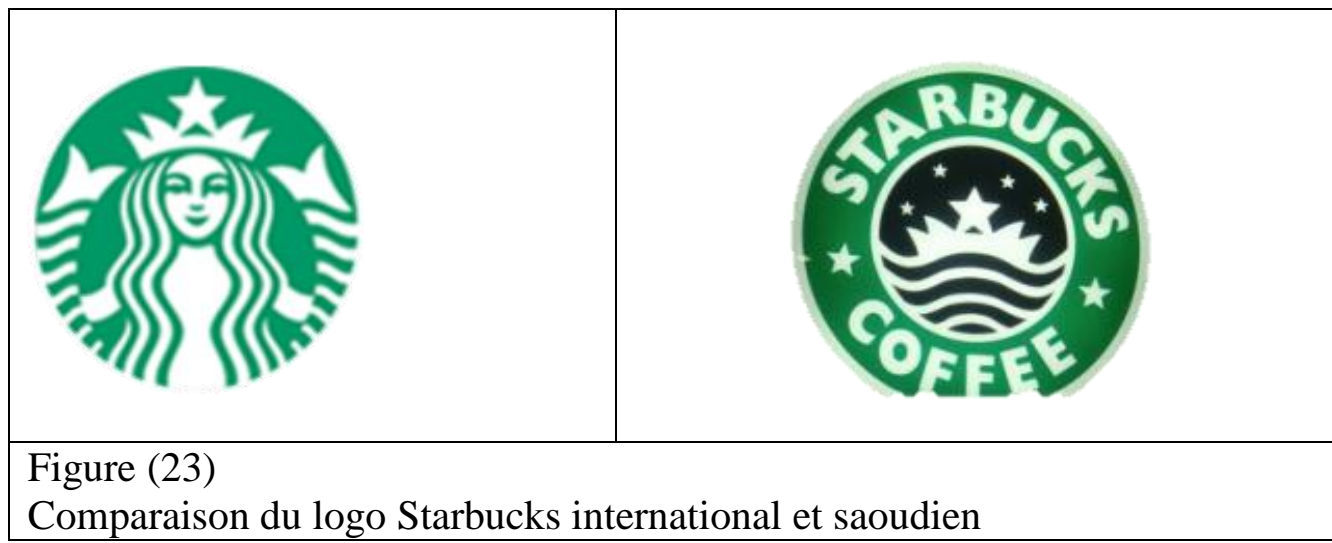

Citons également l'exemple du catalogue d'Ikea ${ }^{\mathbf{5 8}}$ distribué en Arabie Saoudite où les femmes avaient été "effacées" de la version originale du catalogue. Ainsi comme le montre l'image ci-dessous, la mère de famille, présente à gauche sur le catalogue distribué en France, a "disparu" à droite sur le catalogue saoudien ${ }^{59}$.

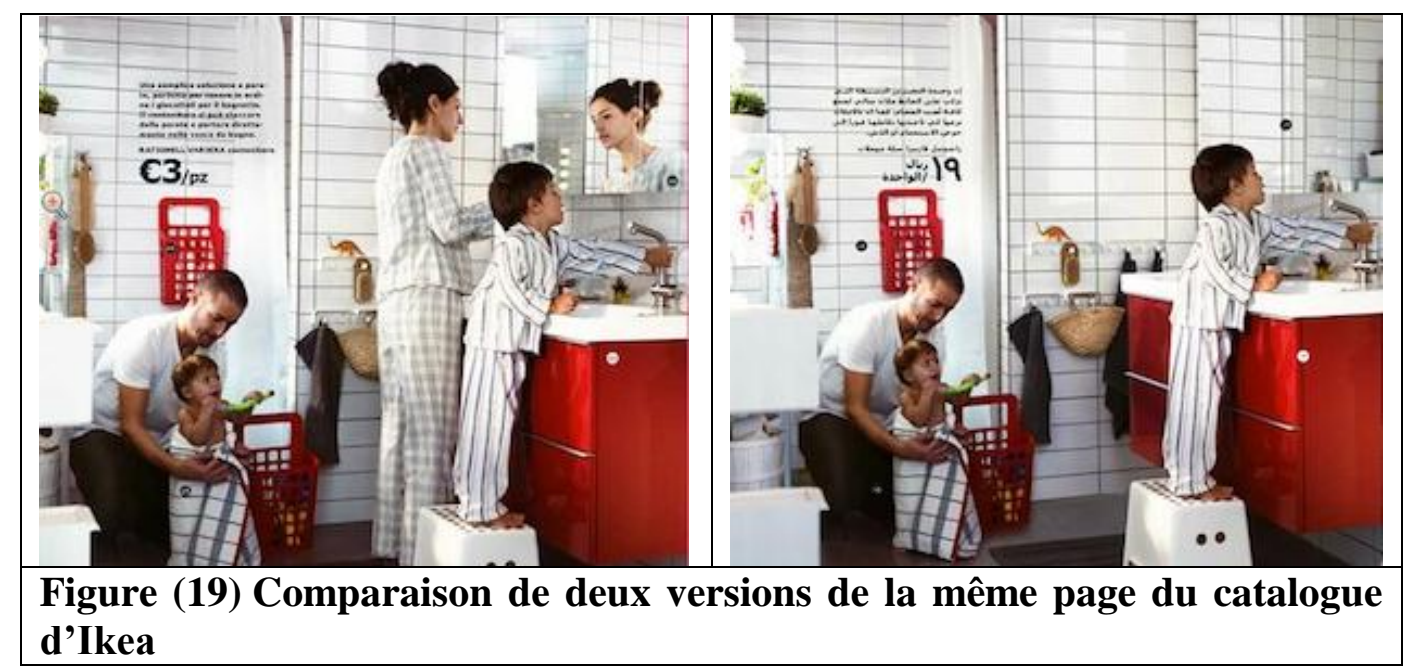

Pour réaliser ces tâches et toucher un public plus large, tout en respectant les normes de la société du pays cible, un traducteur- localisateur doit donc savoir manier les

${ }^{58}$ IKEA, créé en 1943 par Ingvar Kamprad, est une entreprise suédoise dont le siège social se trouve à Delft aux Pays-Bas, spécialisée dans la conception et la vente de détail de mobilier et objets de décoration prêts à poser ou à monter en kit.

${ }^{59}$ https://blogs.mediapart.fr/register/blog/180217/catalogues-ikea-femmes-invisibles-en-israel-et-enarabie-saoudite

Dernière consultation le 02/04/2020

\section{1}


langages informatiques, les formats de fichiers utilisés sur Internet et maintes autres informations, que nous venons de mentionner, concernant la localisation.

\subsection{La localisation des publicités :}

Le troisième type de localisation, autre que celui des logiciels et des sites web, est la localisation publicitaire. En fait, ce troisième type de localisation consiste à adapter linguistiquement et iconographiquement une campagne de communication. En d'autres termes, la localisation des publicités consiste à traduire non seulement le message promotionnel, à savoir : l'appellation (nom de la marque et/ou du produit), le slogan (phrase d'accroche et/ou phrase d'assise), et le rédactionnel (texte d'explication et/ou argumentaire commercial), mais également à effectuer des changements ou des retouches iconographiques dans le but de rendre le même effet sur le public ${ }^{60}$.

Prenons à titre d'exemple la publicité de Pepsi ${ }^{\mathbf{6 1}}$, où le chanteur espagnol de grande renommée, Enrique Iglesias ${ }^{\mathbf{2}}$, présent dans la version américaine ${ }^{63}$, a été remplacé dans la version égyptienne ${ }^{64}$ par Amr Diab ${ }^{65}$, le célèbre chanteur égyptien (Figure 24).

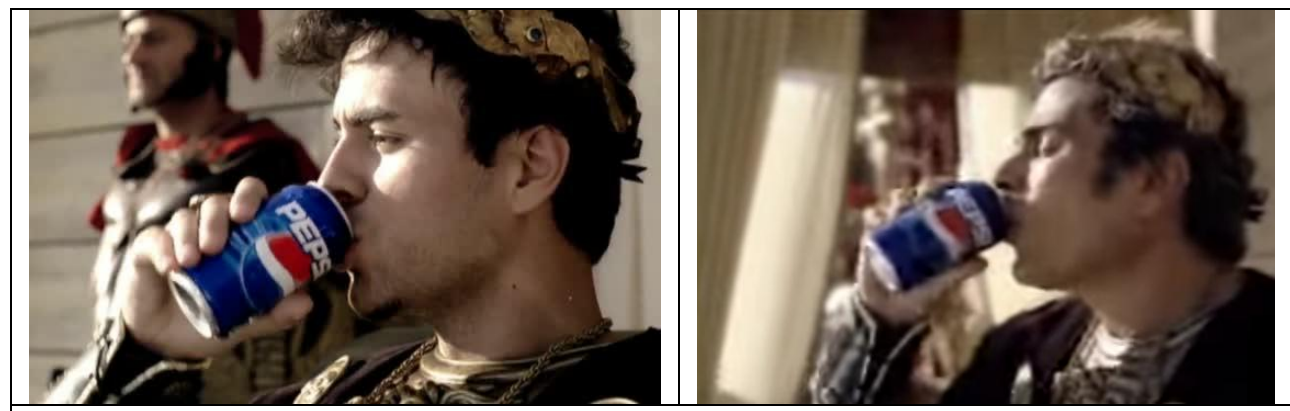

Figure (24) Comparaison de deux versions de la publicité Pepsi

${ }^{60}$ Mathieu GUIDÈRE, « De la traduction publicitaire à la communication multilingue », in Meta, 54 (3), volume 54, Numéro 3, septembre 2009, p.420.

${ }^{61}$ Pepsi est une boisson gazeuse fabriquée par la société PepsiCo qui est une entreprise américaine spécialisée dans le secteur agroalimentaire.

${ }^{62}$ Enrique Iglesias (8 mai 1975) est un auteur, compositeur et chanteur espagnol qui est connu dans en Amérique latine et aux États-Unis.

${ }^{63} \mathrm{https} / / / \mathrm{www}$.youtube.com/watch?v=pES8SezkV8w

Dernière consultation le 10/11/2019

${ }^{64} \mathrm{https}: / /$ www.youtube.com/watch? $\mathrm{v}=2 \mathrm{tPCrin} 1 \mathrm{Rc} 0$,

Dernière consultation le 10/11/2019

${ }^{65}$ Amr Diab (11 octobre 1961) est un chanteur, acteur et compositeur égyptien qui est connu dans le Monde arabe. 


\subsection{La localisation de la documentation technique :}

La localisation de la documentation technique est un processus qui consiste à adapter les manuels d'utilisation ou les modes d'emploi de différents appareils technologiques à un public cible. Ces manuels ou modes d'emploi se composent de textes, de légendes de photos, de captures d'écran, de marqueurs d'index qui doivent tous être bien traduits dans différentes langues et localisés pour différents marchés. Parmi les exemples les plus significatifs, nous pouvons mentionner l'exemple du manuel du téléphone portable Huawei Mate 10 Lite Dans la figure ci-dessous (Figure 25), nous allons voir deux versions de la première page du manuel : une, tirée du manuel français ${ }^{66}$; ; et une autre, tirée du manuel arabe ${ }^{67}$. Nous pouvons constater que les deux versions sont identiques sauf pour la langue et la direction de l'écriture.

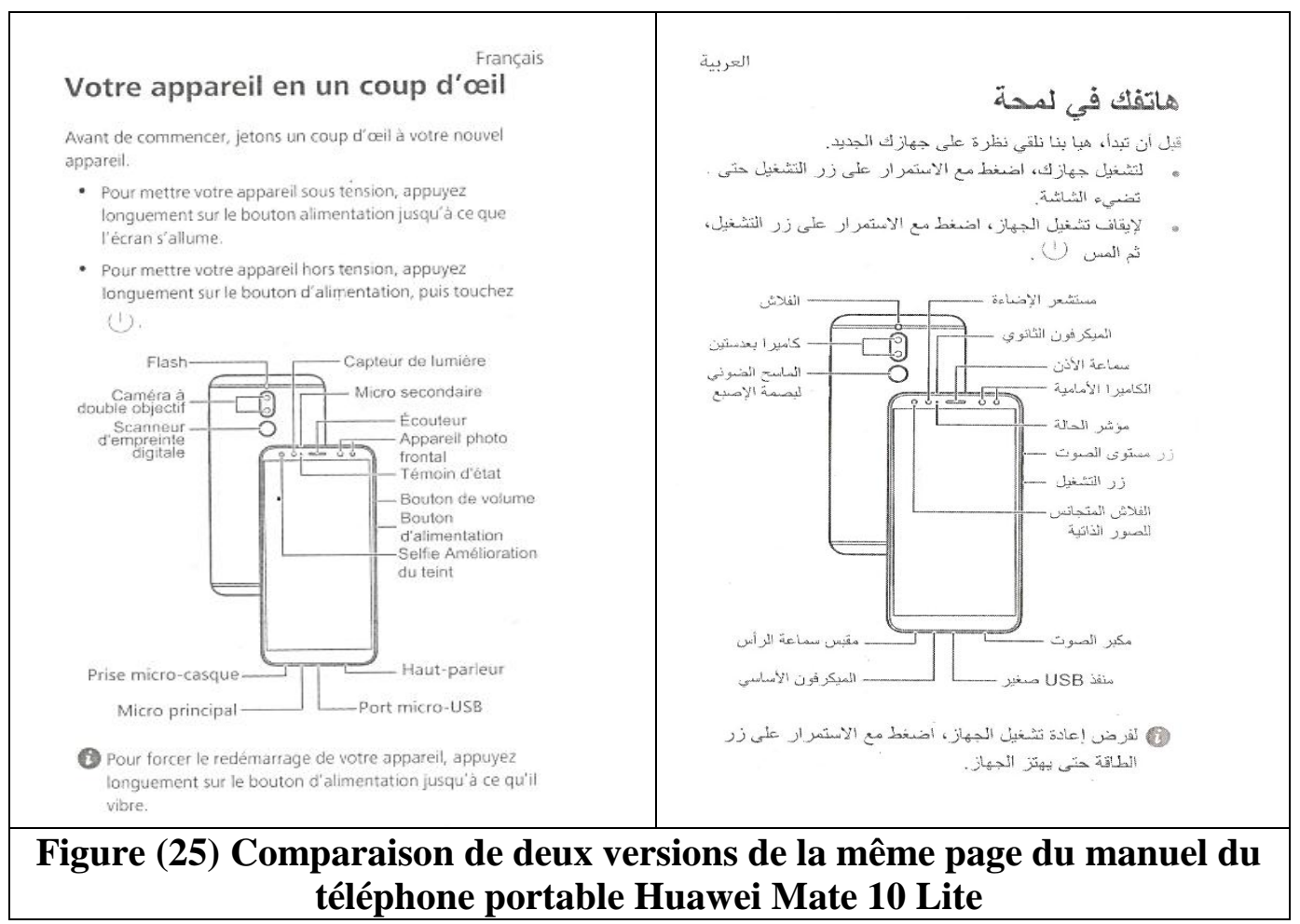

${ }^{66} \mathrm{https}: / /$ mesmodesdemploi.fr/mode-demploi-pour-huawei-mate-10-lite/

Dernière consultation le $05 / 08 / 2020$

${ }^{67} \mathrm{https} / / /$ consumer.huawei.com/sa/support/phones/mate10-lite/

Dernière consultation le 05/08/2020

533 


\subsection{La localisation des multimédias :}

La localisation des multimédias est un processus qui consiste à adapter non seulement des textes écrits, mais aussi du contenu audio et vidéo. Ce contenu multimédia peut prendre la forme de films, de vidéos, de jeux vidéo. Nous avons l'exemple du jeu vidéo FIFA $^{68}$ qui présente, pour la version adaptée au monde arabe, la figure du célèbre défenseur saoudien Yasser Al Shahrani du club Al Hilal Al Saoudi, juste à côté de la figure du défenseur argentin Lionel Messi, de l'équipe Barcelone, mondialement connu et admiré dans le monde arabe (Figure 26).

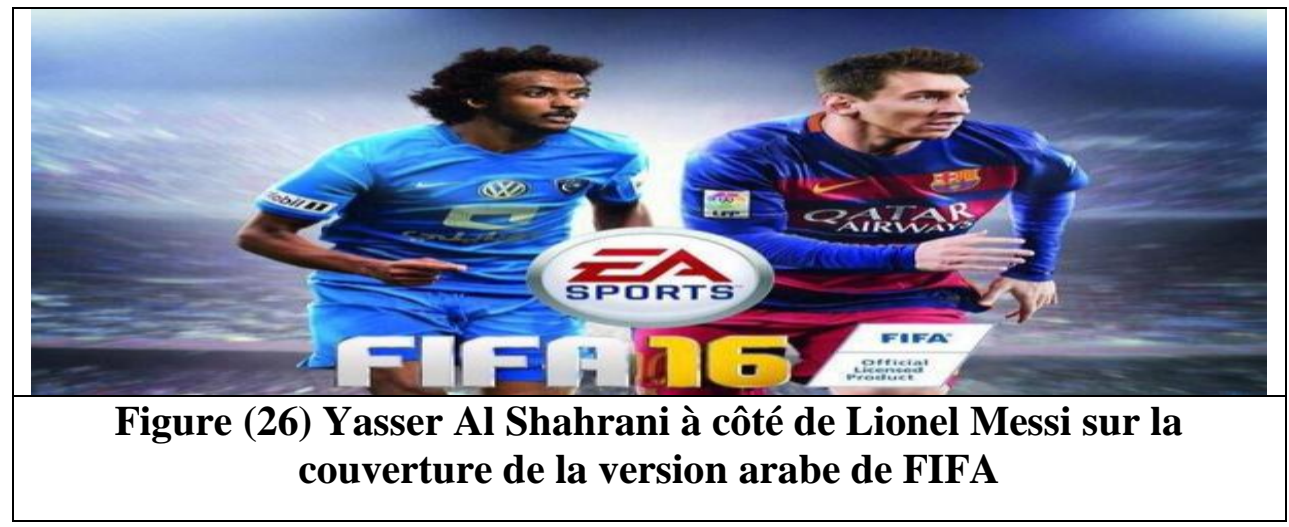

Les localisateurs du jeu ont donc intelligemment choisi d'associer dans une même interface deux figures célèbres dans le monde du football : une, arabe familière à tous les fans de football du Moyen Orient et une figure étrangère, non moins familière pour le monde entier. Par la présence de ces deux figures issues de deux sociétés différentes, sur une même interface, c'est comme si un dialogue interculturel s'établissait de façon implicite entre deux mondes différents.

\subsection{La localisation des produits :}

Dans le domaine de la localisation, il y a également la localisation des produits, à l'exemple du liquide-vaisselle «Persil ». En fait, en France on trouve que ce liquide vaisselle est présenté accompagné de la formule suivante «Soin Noir Baume de Figue ${ }^{69}$, spécialement développée pour préserver l'intensité du noir et des couleurs

\footnotetext{
${ }^{68} \mathrm{https}$ ://fifauteam.com/fifa-16-cover-official-fifa-16-covers/ Dernière consultation le 02/03/2020

${ }^{69} \mathrm{http}: / /$ lemarche-maroc.com/produit.php?prod_id=2215
} 
foncées plus longtemps. Le même produit est présenté dans le monde arabe avec une formule adaptée pour les pays du Golfe : «برسيل شـامبو العبايـان ${ }^{70}$ ou 《Persil shampoing de l'abaya » ${ }^{71}$ et la photo d'une femme portant cette même tenue (Figure 27 ). Pour l'Egypte qui est une société plus ouverte, le produit sera strictement « 》 ou «Persil Noir » ${ }^{72}$ avec deux emballages: le premier, ciblant la grande majorité de la population égyptienne et ayant pour vedette de la publicité, la célèbre «Abla Camel » ${ }^{73}$, actrice égyptienne voilée qui interprète, pour la plupart du temps, des rôles issus de la classe moyenne ou populaire. Le second, affiche la photo d'une femme non voilée, qui n'est autre que la belle vedette égyptienne Ghada $\mathrm{Adel}^{74}$ qui est, quant à elle, une actrice plus moderne et plus sophistiquée par ses rôles et sa tenue (Figure 28).

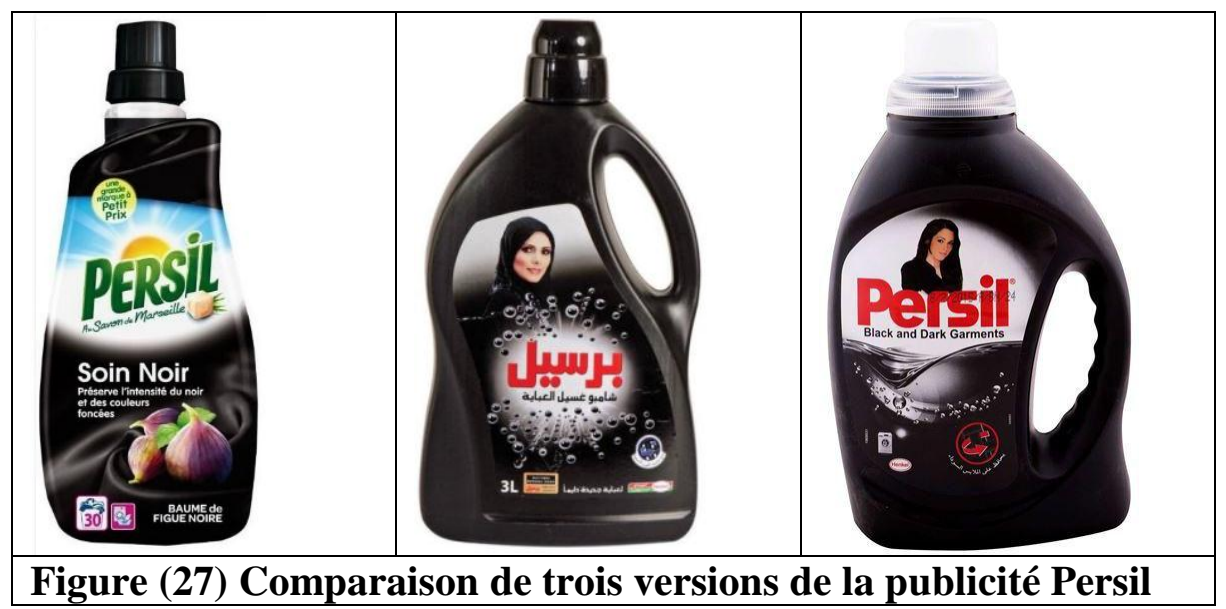

Dernière consultation le 02/03/2020

${ }^{70}$ https://www.othaimmarkets.com/home-care/household/persil-abaya-shampoo-31.html

Dernière consultation le 02/03/2020

${ }^{71} \mathrm{~L}$ 'abaya est un vêtement long, ample et fluide apprécié par les femmes musulmanes.

72 https://wengz.net/index.php?route=product/product\&product $\mathrm{id}=726$ Dernière consultation le 02/03/2020

73 https://www.youtube.com/watch?v=oBElQKC48Bc

Dernière consultation le $02 / 03 / 2020$

${ }^{74}$ https://www.youtube.com/watch?v=Hj0cPeH2CnY

Dernière consultation le 02/03/2020

535 


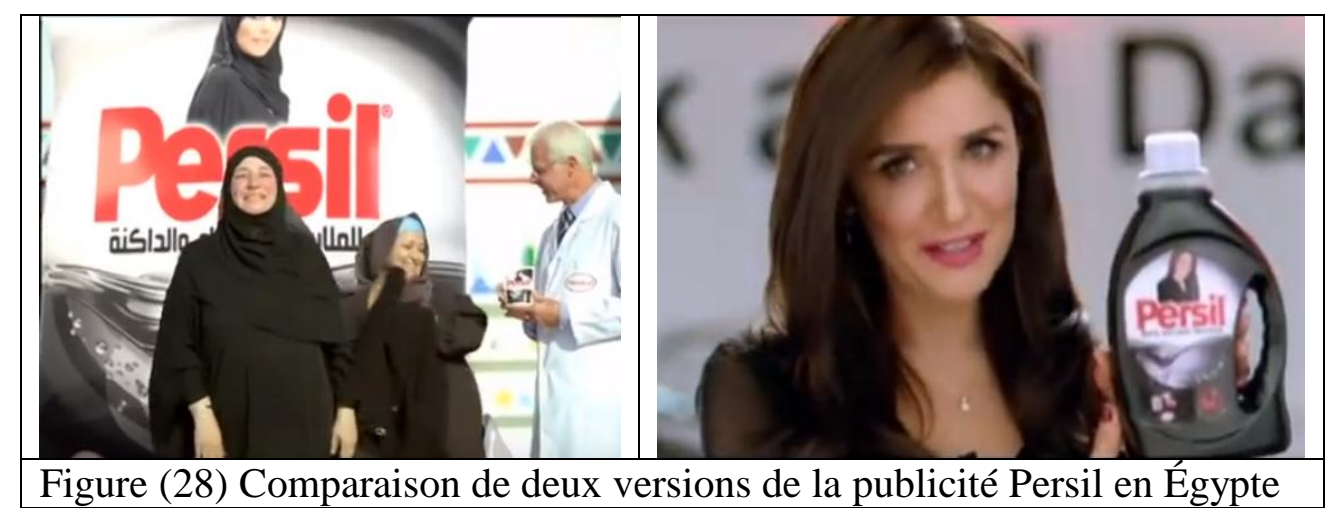

Le localisateur du produit a tenu compte des différence socio-culturelles entre les deux communautés, française et égyptienne, ainsi que de leurs besoins. Ajoutons également que le travail du localisateur est allé plus loin encore puisqu'il a tenu compte des différences socio-culturelles existantes à l'intérieur d'une même communauté avec le choix de Abla Camel et de Ghada Adel. Par ces choix pertinents, le localisateur a réussi ainsi à entretenir un dialogue interculturel avec le public ciblé par ces produits.

\section{-Conclusion :}

Tout ce qui précède nous mène à la constatation que la localisation, en tant qu'adaptation de produits, d'instruments, de concepts et de structures à un 'locus" particulier de la planète ou à des conditions locales, joue un rôle de première importance dans le dialogue interculturel. En fait, à l'ère de l'informatique et d'Internet, toutes les sociétés s'attendent à ce que toute information au sujet des produits et des services soit donnée dans leur propre langue ou une langue qu'ils comprennent.

Il est ainsi primordial de promouvoir cette diversité non seulement pour garantir une plus grande compétitivité des produits et des services mais surtout pour éviter l'établissement d'une culture mondiale unique ou d'un malentendu culturel. En d'autres termes, la localisation est importante :

«Si l'on veut que les populations des pays les plus pauvres aient un meilleur accès aux technologies de l'information et de la communication, que ces technologies correspondent davantage aux vies, aux besoins et aux aspirations de ces populations 
et, en bout de ligne, que soit réduite la fracture numérique $\gg{ }^{75}$.

Par les exemples que nous avons choisis pour chaque type de localisation, et l'approche comparative que nous avons adoptée, nous avons tenté de prouver que la localisation, malgré ses multiples enjeux, joue un rôle primordial dans la réduction de l'écart interculturel. Les produits localisés aident ainsi à établir une sorte de dialogue avec le public pour lequel ils ont été localisés puisqu'ils s'adressent à ce public dans sa propre langue et en revêtant les caractéristiques qui lui sont propres.

Dans cette perspective d'une approche interculturelle, et surtout à l'époque de la globalisation et de la perception de l'hybridité des civilisations, il parait donc important de ne pas succomber à la tentation d'un nivelage des particularités culturelles, à l'illusion d'une compréhension de l'altérité ainsi qu'à l'idée d'une cohabitation multiculturelle sans problème. Il s'agit plutôt de se rendre sensible aux spécifiés de l'étrangeté de l'autre, en affirmant cette étrangeté, et non pas en l'ignorant ou en la nivelant. Une des tâches primordiales du localisateur devrait, en ce sens, consister en une approche qui fasse preuve de sa sensibilité à la différence de l'étranger et de son respect pour cette différence.

${ }^{75}$ Don OSBORN, Les Langues Africaines à l'ère du numérique : défis et opportunités de l'informatisation des langues autochtones, Traduit de l'anglais par Geneviève Deschamps, PUL, Centre de recherches pour le développement international, 2011, préface.

\section{7}




\section{- Bibliographie}

\section{Ouvrages consultés :}

\section{Ouvrages généraux :}

-Don OSBORN, Les Langues Africaines à l'ère du numérique: défis et opportunités de l'informatisation des langues autochtones, Traduit de l'anglais par Geneviève Deschamps, PUL, Centre de recherches pour le développement international, 2011, préface.

2.Ouvrages consacrés à la traductologie et la linguistique :

-BARTHES, Roland: Essais critiques, Paris, Editions du Seuil, Coll. "Telque", 1964, 278p.

- CHESTERMAN, A, Memes of Translation. The Spread of Ideas in Translation Theory, Amsterdam/Philadelphia, John Benjamins, coll. «Benjamins Translation Library $22 »,(2000)$.

-DELISLE, Jean, La Traduction raisonnée : manuel d'initiation à la traduction professionnelle de l'anglais vers le français., Les Presses de l'Université d'Ottawa, 1993.

-GUIDERE, Mathieu, La traduction arabe, Méthodes et applications, De la traduction à la traductique, ellipses, 2005.

-MONTRUEUX, Marie-Françoise, La lexicologie entre langue et discours, Armand Colin, Campus, Paris, 2004, 192 p.

-REISS, Katharina, Problématiques de la traduction, éds. 'Ecnomica, Coll. Bibliothèque de traductologie, Paris, 2009, $200 \mathrm{p}$.

- $\quad$ VINAY, J.-P. et DARBELNET, J., Stylistique comparée du Français et de l'Anglais, Didier, 2004.

3. Ouvrages consacrés à la traduction multimédias :

-CORNU, Jean-François : Le doublage et le sous- titrage : Histoire et esthétique, 2014.

-ESSELINK, Bert A practical guide to localization, Amsterdam/Philadelphia, John Benjamins Publishing Company, 2000.

-LE NOUVEL, Thierry : Le Doublage, Eyrolles, Ciné-métiers, 2007 
-MAROTO, Jésus et DE BORTOLI, Mario, Web Site Localisation, European Languages and the Implementation of Communication and Information Technologies (Elicit) Conférence, 2001.

-PETTIT, Zoé , Sous-titrage et doublage : interaction entre langage et image dans une sélection de genres audiovisuels, soutenue à Montpellier 3, Thèse sous la direction de Jacky MARTIN, 2000.

-SCHÄLER Reinhard, Localization dans Mona BAKER et Gabriela SALDANHA, Routledge encyclopedia of translation studies, 2ème edition, Abingdon, Routledge, 2009.

4.Ouvrages consacrés à l'interculturel :

-LADMIRAL, Jean - René et LIPIANSKY, Edmond Marc, La Communication interculturelle, Coll. Traductologie, Les Belles Lettres, 2015, p. 97.

\section{II.Articles consultés :}

1. Articles généraux :

-FOUILLEE, Alfred, «Psychologie de l'Esprit français autrefois et aujourd'hui », in Revue des Deux Mondes, 4e période, tome 138, 1896, p.66.

2.Articles consacrés à la traduction multimédias :

-Daniel GOUADEC, «Le bagage spécifique du localiseur/localisateur : Le vrai nouveau profil requis », in Meta Volume 48, Issue 4, Décembre 2003, p.126.

-ALSHTAIWI, Ma'moun, «Parlons du localisateur : rôle et profil », in Traduire, n. 237, 2017, pp.38-47.

\section{Webographie}

\section{I.Dictionnaires en ligne :}

-http://www.granddictionnaire.com/ficheOqlf.aspx?Id_Fiche=506326

Dernière consultation le 5 avril 2018

-https://www.larousse.fr/dictionnaires/francais/localisation/47591?q=la+localisa tion\#47516

Dernière consultation le 7 avril 2018

معجم لسان العرب لابن منظور (المعجم الالكتروني)-

http://shamela.ws/index.php/book/1687

معجم المعاني (المعجم الالكتروني)-

http://www.almaany.com 


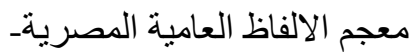

http://dar.bibalex.org/webpages/mainpage.jsf?PID=DAF-Job:179886

\section{II.Ouvrage en ligne :}

- DURDURE ANU, Ioana Irina, «Traduction et typologie des textes», in http://ler.letras.up.pt/uploads/ficheiros/9808.pdf, page 5.

Dernière consultation le 2 février 2016

\section{III.Articles en ligne :}

-«Localisation et traduction : quelles différences ? », in https://culturesconnection.com/fr/localisation-traduction-differences/

Dernière consultation le 8 juillet 2019.

\section{IV.Sites :}

-http://www.jeuneafrique.com/102196/archives-thematique/egypte-fulla-contrebarbie/

Dernière consultation le 30/12/2017. -https://www.djazairess.com/fr/elwatan/103452,

Dernière consultation le 30/12/2017. -https://cris23.fr/calendriers.htm

Dernière consultation le 09 janvier 2018. -https://www.apbif.fr/noms-des-mois-lunaires/

Dernière consultation le 09 janvier 2018.

-https://tresoreducatif.com/les-mois-de-lannee-calendrier-lunaire-et-solaireoriental-et-occidental/,

Dernière consultation le 09 janvier 2018.

-https://frenchmorning.com/pourquoi-les-americains-mettent-le-mois-en-tete-de-

la-date/. Dernière consultation le 19 janvier 2018. -http://www.lesmetiers.net/orientation/p1_197405/les-metiers-de-la-traduction

Dernière consultation le 06/06/ 2018. -https://www.gala-global.org/

Dernière consultation le 10/10/2018 -https://www.elocalize.net/

Dernière consultation le 12/10/2018 -http://skylingos.com/

Dernière consultation le 12/10/2018 -https://arabization-translation.net/

Dernière consultation le 12/10/2018 -http://www.arabize.com/

Dernière consultation le 12/10/2018 -http://www.sdltrados.com/fr/solutions/software-localization/

Dernière consultation le 05/12/2018. -https://www.youtube.com/watch?v=wYQKgCcMqZY pour la version arabe. 
Dernière consultation le 08/07/ 2019.

-https://www.youtube.com/watch?v=45y8q2h1HGI pour la version française

Dernière consultation le 08/07/2019.

-https://www.youtube.com/watch?v=pES8SezkV8w

Dernière consultation le 10/11/2019

-https://www.youtube.com/watch?v=2tPCrin1Rc0,

Dernière consultation le 10/11/2019

-https://fifauteam.com/fifa-16-cover-official-fifa-16-covers/

Dernière consultation le 02/03/2020

-http://lemarche-maroc.com/produit.php?prod_id=2215

Dernière consultation le 02/03/2020

-https://support.minitab.com/fr-fr/minitab/18/help-and-how-to/manipulate-data-

in-worksheets-columns-and-rows/supporting-topics/date-time-data-and-

formats/defining-custom-date-time-display-formats/

Dernière consultation le 02/04/2020

-https://blogs.mediapart.fr/register/blog/180217/catalogues-ikea-femmes-

invisibles-en-israel-et-en-arabie-saoudite

Dernière consultation le 02/04/2020

-https://www.egyptair.com/en/Pages/HomePage.aspx

Dernière consultation le 02/06/2020

-https://www.egyptair.com/ar/contact-us/Pages/DomesticOffices.aspx

Dernière consultation le 02/06/2020

-https://mesmodesdemploi.fr/mode-demploi-pour-huawei-mate-10-lite/

Dernière consultation le 05/08/2020

-https://consumer.huawei.com/sa/support/phones/mate10-lite/

Dernière consultation le 05/08/2020 